University of Nebraska - Lincoln

DigitalCommons@University of Nebraska - Lincoln

4-1-1995

\title{
Resonantly laser-driven plasma waves for electron acceleration
}

Donald P. Umstadter

University of Nebraska-Lincoln, donald.umstadter@unl.edu

J. Kim

University of Michigan - Ann Arbor

Eric Esarey

Plasma Physics Division, Naval Research Laboratory, Washington, D.C., ehesarey@lbl.gov

E. Dodd

University of Michigan - Ann Arbor

T. Neubert

University of Michigan - Ann Arbor

Follow this and additional works at: https://digitalcommons.unl.edu/physicsumstadter

Part of the Physics Commons

Umstadter, Donald P.; Kim, J.; Esarey, Eric; Dodd, E.; and Neubert, T., "Resonantly laser-driven plasma waves for electron acceleration" (1995). Donald Umstadter Publications. 10.

https://digitalcommons.unl.edu/physicsumstadter/10

This Article is brought to you for free and open access by the Research Papers in Physics and Astronomy at DigitalCommons@University of Nebraska - Lincoln. It has been accepted for inclusion in Donald Umstadter Publications by an authorized administrator of DigitalCommons@University of Nebraska - Lincoln. 


\title{
Resonantly laser-driven plasma waves for electron acceleration
}

\author{
D. Umstadter, ${ }^{1,2}$ J. Kim, ${ }^{1}$ E. Esarey, ${ }^{1,3}$ E. Dodd, ${ }^{1}$ and T. Neubert ${ }^{1,4}$ \\ ${ }^{1}$ Center for Ultrafast Optical Science, University of Michigan, Ann Arbor, Michigan 48109 \\ ${ }^{2}$ Electrical Engineering and Computer Science Department, University of Michigan, Ann Arbor, Michigan 48109 \\ ${ }^{3}$ Plasma Physics Division, Naval Research Laboratory, Washington, D.C. 20375 \\ ${ }^{4}$ Space Physics Research Laboratory, University of Michigan, Ann Arbor, Michigan 48109
}

(Received 8 November 1994)

\begin{abstract}
A method for generating large-amplitude nonlinear plasma waves, which utilizes an optimized train of independently adjustable, intense laser pulses, is analyzed in one dimension both theoretically and numerically (using both Maxwell-fluid and particle-in-cell codes). Optimal pulse widths and interpulse spacings are computed for pulses with either square or finite-rise-time sine shapes. A resonant region of the plasma wave phase space is found where the plasma wave is driven most efficiently by the laser pulses. The width of this region, and thus the optimal finite-rise-time laser pulse width, was found to decrease with increasing background plasma density and plasma wave amplitude, while the nonlinear plasma wavelength, and thus the optimal interpulse spacing, increases. Also investigated are damping of the wave by trapped background electrons and the sensitivities of the resonance to variations in the laser and plasma parameters. Resonant excitation is found to be superior for electron acceleration to either beat-wave or single-pulse excitation because comparable plasma-wave amplitudes may be generated at lower plasma densities, reducing electron-phase detuning, or at lower laser intensities, reducing laser-plasma instabilities. Practical experimental methods for producing the required pulse trains are discussed.
\end{abstract}

PACS number(s): 52.75.Di, 52.40.Nk, 52.35.Mw

\section{INTRODUCTION}

The generation of large-amplitude, relativistic plasma waves is a subject of much current interest [1] because of its potential use for ultrahigh-gradient electron acceleration [2]. While conventional rf-driven accelerators are limited to fields $\leq 1 \mathrm{MV} / \mathrm{cm}$, plasma accelerators have been shown experimentally to support gradients $\leq 100$ $\mathrm{MV} / \mathrm{cm}$ [3]. The maximum axial electric field of a relativistic plasma wave, as predicted by one-dimensional (1D) cold fluid theory, is the "wave-breaking" field [4], $E_{\mathrm{WB}}=E_{0} \sqrt{2\left(\gamma_{p}-1\right)}$, where [5] $E_{0}=\left(m_{e} c \omega_{p} / e\right) \simeq$ $0.96 n_{e 0}^{1 / 2}\left[\mathrm{~cm}^{-3}\right] \mathrm{V} / \mathrm{cm}, \omega_{p}=\left(4 \pi e^{2} n_{e 0} / m_{e}\right)^{1 / 2}$ is the electron plasma frequency, $n_{e 0}$ is the ambient electron density, $\gamma_{p}=\left(1-v_{p}^{2} / c^{2}\right)^{-1 / 2}$, and $v_{p}$ is the phase velocity of the plasma wave. For a laser-driven, plasma-based accelerator, $\gamma_{p} \simeq \gamma_{g} \simeq \omega / \omega_{p} \gg 1$, where $\gamma_{g}=\left(1-v_{g}^{2} / c^{2}\right)^{-1 / 2}$, $v_{g}$ is the group velocity of the laser, and $\omega$ is the laser frequency. For a laser of wavelength $\lambda \simeq 2 \pi c / \omega=1 \mu \mathrm{m}$ and a plasma of density $n_{e 0}=10^{16} \mathrm{~cm}^{-3}, \gamma_{g} \simeq 300$, $E_{0} \simeq 100 \mathrm{MV} / \mathrm{cm}$, and $E_{\mathrm{WB}} \simeq 2.5 \mathrm{GV} / \mathrm{cm}$.

Until recently, only two major types of laser-driven, plasma-based accelerators had been investigated: the plasma beat-wave accelerator (PBWA) $[2,3]$ and the laser wake-field accelerator (LWFA) $[2,6-8]$. In the PBWA, two laser beams of frequencies $\omega_{1}$ and $\omega_{2}$ are optically mixed in a plasma to produce a laser beat wave of frequency $\Delta \omega=\omega_{1}-\omega_{2}$, i.e., in effect a train of fixed equally spaced pulses of equal pulse widths. By adjusting the beat frequency and/or the plasma density such that $\Delta \omega \simeq \omega_{p}$, the laser beat wave can resonantly drive a large-amplitude plasma wave. As the plasma-wave am- plitude grows, however, nonlinear effects cause the wavelength of the plasma wave to increase and, hence, the resonant frequency is shifted away from $\Delta \omega$. Eventually the relative phase between the laser beat wave and the plasma wave becomes $\pi / 2$ out of phase and the beat wave no longer drives the plasma wave to higher amplitudes. This resonance detuning due to the increase in the plasma wavelength ultimately limits the plasma-wave amplitude in the PBWA. In the LWFA, the ponderomotive force associated with the gradients in the intensity of a single, ultrashort laser pulse [9] drives a plasma-wave "wake field." The maximum plasma-wave amplitude is obtained when the pulse duration $\tau$ and/or plasma density is adjusted such that $\tau \simeq 2 \pi / \omega_{p}$. The plasma-wave amplitude increases as the laser intensity $I$ increases and the laser pulse length decreases (i.e., as the density increases). Hence, in the LWFA, the plasma-wave amplitude is limited by the maximum laser intensity and the minimum laser pulse length that can be obtained by laser technology. Currently, these values are limited to $I \sim 10^{18} \mathrm{~W} / \mathrm{cm}^{2}$ and $\tau \sim 50 \mathrm{fs}[9]$.

Recently, the self-modulated LWFA has been suggested $[10,11]$. Here, a single laser pulse is incident on a plasma with a density that is higher than the "resonant density" such that the laser pulse duration is now several plasma periods, i.e., $\tau>2 \pi / \omega_{p}$. Due to a self-modulation instability [12], the pulse breaks up into multiple pulses, each of which is "resonant." Although higher plasma densities and the high-intensity multiple-pulse structure lead to higher wake-field amplitudes, they are difficult to achieve simultaneously due to plasma defocusing [13]. In simulations of the self-modulated LWFA [10], the electron energy gain was observed to be limited by phase detuning 
between the accelerated electrons and the plasma wave.

Electron-phase detuning is a fundamental limitation in all plasma-based accelerators, i.e., accelerated electrons (with $v \rightarrow c$ ) outrun the plasma wave (with $v_{p} \simeq v_{g}<c$ ). Acceleration will cease once the electrons phase advance a distance $\left(v-v_{p}\right) t \simeq \lambda_{p} / 2$ relative to the plasma wave, where $\lambda_{p}=2 \pi c / \omega_{p}$ is the plasma-wave length. In the laboratory frame, this corresponds roughly to a detuning distance of $L_{t} \simeq \gamma_{p}^{2} \lambda_{p}$, where $v=c$ has been assumed. It can be shown [14] that the maximum energy gain $\Delta W_{t}$ of a trapped electron in a 1D plasma wave of amplitude $E_{z}$ is $\Delta W_{t} \simeq 4 m_{e} c^{2} \gamma_{p}^{2} E_{z} / E_{0}$ for $E_{z}^{2} / E_{0}^{2} \ll 1$, and in the nonlinear limit, $\Delta W_{t} \simeq 2 m_{e} c^{2} \gamma_{p}^{2}\left(E_{z} / E_{0}\right)^{2}$ for $E_{z}^{2} / E_{0}^{2} \gg 1$ [14]. For example, for a fixed value of $\epsilon=E_{z} / E_{\mathrm{WB}}=0.25$ and a laser wavelength of $\lambda=1 \mu \mathrm{m}$, $\Delta W_{t} \simeq 4.6 \mathrm{GeV}$ for $n_{e 0}=10^{18} \mathrm{~cm}^{-3}\left(E_{\mathrm{WB}}=7.7\right.$ $\mathrm{GV} / \mathrm{cm})$, whereas $\Delta W_{t} \simeq 4.6 \mathrm{TeV}$ for $n_{e 0}=10^{16} \mathrm{~cm}^{-3}$ $\left(E_{\mathrm{WB}}=2.5 \mathrm{GV} / \mathrm{cm}\right)$, where $\gamma_{p} \simeq \omega / \omega_{p} \gg 1$ has been assumed. Notice that for a fixed $\epsilon, \Delta W_{t} \simeq 4 m_{e} c^{2} \gamma_{p}^{3} \epsilon^{2} \sim$ $n_{e 0}^{-3 / 2}$, assuming $E_{z}^{2} / E_{0}^{2} \gg 1$ and $\gamma_{p}^{2} \gg 1$. Hence, at the high densities required either for self-modulation or for the use of an ultrashort pulse in the standard LWFA, $\gamma_{g}$ is relatively low and acceleration is limited by electronphase detuning. As will be discussed below, the acceleration at high density is limited not only by phase detuning but by the pump depletion length $L_{d}$.

In a previous paper [15], we proposed an alternative accelerator concept, which we call the resonant laserplasma accelerator (RLPA), that combines the virtues of these others, but has the following additional advantages: (i) by utilizing a train of laser pulses with independently adjustable pulse widths and interpulse spacings, which are varied in an optimized manner, resonance with both the changing plasma-wave period and phase resonance width can be maintained in the nonlinear regime, and the maximum plasma-wave amplitude is achieved; (ii) lower plasma densities can be used, thus avoiding electron-phase detuning; and (iii) lower peak laser intensities can be used, thus allowing for a reduction of laser-plasma instabilities. In this paper we investigate the RLPA concept [15-17] in greater detail, including important issues such as (1) the sensitivities of the wake field to changes in the laser and plasma parameters, (2) comparisons with the PBWA and LWFA, and (3) damping of the wave by trapped electrons.

This paper is concerned with determining the characteristics of the plasma wave generated by a nonevolving, optimized laser pulse train in 1D. The laser intensity profile of the pulse train is assumed to be nonevolving and a function of only the variable $\zeta=v_{p} t-z$, where $z$ is the axial propagation distance and $v_{p}=v_{g}$ is assumed. Neglected are various effects that could degrade the evolution of the laser pulse train, such as diffraction, pump depletion, and laser-plasma instabilities.

In the absence of some form of optical guiding, a laser pulse will diffract after propagating a distance characterized by the Rayleigh length, $Z_{R}=\pi r_{0}^{2} / \lambda$, where $r_{0}$ is the minimum laser spot size at focus. For a tightly focused laser pulse, $Z_{R}$ can be relatively short. However, it has been observed both numerically [10] and experimentally [18] that a preformed plasma channel can be used to guide the laser pulse and prevent diffraction.

Pump depletion refers to the process by which the laser pulse loses energy as it generates a plasma wave. A rough estimate for the pump depletion length $L_{d}$ is given by equating the energy left behind in the plasma wave to the initial energy in the laser pulse train, i.e., $L_{d} E_{z}^{2} \simeq c \tau_{\text {tot }} E_{L}^{2}$. Here, it is assumed that the laser pulse train consists of pulses with equal intensities $\left(E_{L}\right.$ is the amplitude of the electric field of the laser pulse) and the sum of the pulse durations is $\tau_{\text {tot }}$. As an example, consider the sine pulse train described in Sec. IIIB. Here, $E_{L}=38 \mathrm{GV} / \mathrm{cm}, \tau_{\text {tot }}=2.2 \mathrm{ps}$, and $E_{z}=0.18 \mathrm{GV} / \mathrm{cm}$, which implies a depletion length of $L_{d}=30 \mathrm{~m}$. In order to achieve large energy gains $\Delta W$, large pump depletion distances must also be obtained $\Delta W=z E_{z} \leq L_{d} E_{z}$, where $z$ is the acceleration length. In the limit $\gamma_{g} \gg 1$, the energy gain after a pump depletion length scales roughly as one over the density, i.e., $L_{d} E_{z} \sim 1 / n_{e 0}$. Hence, for high-energy gains, operating at lower plasma densities is required in order to avoid pump depletion, just as with phase detuning. To obtain energy gains larger than the pump depletion limit, multiple acceleration stages appear necessary. Alternatively, the use of an active medium has been suggested as a method to overcome pump depletion [19].

Several laser-plasma instabilities could degrade the laser pulses as they propagate, such as stimulated Raman scattering, modulation and filamentation instabilities, and parametric coupling to ion modes. Typically, the growth rates of these instabilities increase with increasing laser intensity. Since the use of multiple laser pulses reduces the peak intensity required to drive a large-amplitude plasma wave, the growth rates for the various instabilities can be reduced. Furthermore, the analyses of these various instabilities generally assume long uniform pulse profiles. It is not clear how a pulse train structure will affect the behavior of the instabilites. It may also be possible to reduce and/or eliminate some instabilities by introducing pulse-to-pulse phase incoherence of the high-frequency laser oscillations within the train.

The analysis and numerical examples presented in this paper are based on a $1 \mathrm{D}$ model. The validity of the $1 \mathrm{D}$ model of plasma-wave excitation requires that $d_{0}>\lambda_{p}$, where $d_{0}=2 r_{0}$ is the transverse laser spot diameter. The above discussions on electron-phase detuning and pump depletion indicate that in order to achieve large single stage energy gains, low plasma densities are preferred. To achieve high laser intensities at low plasma densities while remaining in the $1 \mathrm{D}$ limit implies ultrahigh laser powers. For example at $n_{e 0}=10^{16} \mathrm{~cm}^{-3}, \lambda_{p}=330 \mu \mathrm{m}$, and to obtain an intensity of $I \simeq 10^{18} \mathrm{~W} / \mathrm{cm}^{2}$ requires a laser power of $P \geq I \pi\left(d_{0} / 2\right)^{2} \simeq 850 \mathrm{TW}$, assuming $d_{0} \geq \lambda_{p}$. Such powers can be obtained by the petawatt $\left(10^{15} \mathrm{~W}\right)$ laser systems currently under construction at Lawrence Livermore National Laboratory [20] and elsewhere. Proof-of-principle experiments, however, can be performed in the 1D limit at higher plasma densities and lower intensities with smaller but shorter pulse laser systems. For example, at $n_{e 0}=5 \times 10^{17} \mathrm{~cm}^{-3}, \lambda_{p}=47 \mu \mathrm{m}$, and an intensity of $I \simeq 5 \times 10^{17} \mathrm{~W} / \mathrm{cm}^{2}$ requires $P \geq 8.5$ 
TW, which can be obtained with state-of-the-art "tabletop" systems.

In Sec. II, analytic solutions and sample calculations for a square pulse train are presented to demonstrate the advantage of the use of multiple pulses in terms of amplitude and energy efficiency. In Sec. III, numerical results are presented of pulse trains of both the square and sine shapes. Comparison of the different acceleration schemes suggests that the RLPA is more efficient than either the PBWA or the LWFA (Sec. III B 2). We also discuss the sensitivities of the wake fields produced by the various plasma-wave generation schemes to changes in the laser and plasma parameters, such as laser intensity (Sec. III B 6), laser pulse widths and interpulse spacings (Sec. III B 4), and plasma density (Sec. III B 5). Kinetic effects investigated by use of a particle-in-cell (PIC) code simulation are discussed in Sec. IV. Pulse-shaping techniques are discussed in Sec. V. Conclusions are presented in Sec. VI.

\section{ANALYSIS}

The laser-plasma interaction is modeled by the relativistic Maxwell-fluid equations. The laser pulse is described by the normalized transverse vector potential, $\vec{a}=e \vec{A}_{\perp} / m_{e} c^{2}$. The laser envelope $|a|$ is assumed to be nonevolving and a function of only $\zeta=v_{g} t-z$, where $v_{g}$ is the group velocity (assumed constant), i.e., the "quasistatic" approximation [8]. Circular polarization is assumed, i.e., $a^{2}=a^{2}(\zeta)$. The quantity $a^{2}$ is related to the laser wavelength $\lambda$ and intensity $I$ by $a \simeq 6 \times 10^{-10} \lambda[\mu \mathrm{m}] I^{1 / 2}\left[\mathrm{~W} / \mathrm{cm}^{2}\right]$. The plasma response is described by the normalized electrostatic potential, $\phi=e \Phi / m_{e} c^{2}$, which in the 1D limit obeys the nonlinear Poisson equation $[15-17,21]$

$$
\frac{d^{2}}{d \zeta^{2}} \phi=k_{p}^{2} \gamma_{g}^{2}\left[\beta_{g}\left(1-\frac{\left(1+a^{2}\right)}{\gamma_{g}^{2}(1+\phi)^{2}}\right)^{-1 / 2}-1\right]
$$

where $\beta_{g}=v_{g} / c, \gamma_{g}=\left(1-\beta_{g}^{2}\right)^{-1 / 2}$, and $k_{p}=\omega_{p} / c$ is the plasma wave number. In deriving Eq. (1), $\phi$ was assumed to be a function of only $\zeta$, i.e., $v_{p} \simeq v_{g}$. In the limit $a^{2} \ll 1, \gamma_{g}=\omega / \omega_{p}$ (nonlinear corrections are discussed in [22]). As previously mentioned, the laser pulse structure is assumed to be nonevolving. This ignores various effects, such as diffraction, pump depletion, and laser-plasma instabilities.

\section{Square pulses}

Several properties of the plasma wave can be determined analytically from Eq. (1) for a series of square laser pulses. When $a^{2}$ is constant, Eq. (1) can be integrated to yield

$$
\begin{aligned}
x^{\prime 2}= & 2 \gamma_{g}^{2}\left\{\left(x_{0}-x\right)\right. \\
& \left.+\beta_{g}\left[\left(x^{2}-\gamma_{\perp}^{2} / \gamma_{g}^{2}\right)^{1 / 2}-\left(x_{0}^{2}-\gamma_{\perp}^{2} / \gamma_{g}^{2}\right)^{1 / 2}\right]\right\},
\end{aligned}
$$

where $x=1+\phi, \gamma_{\perp}=\left(1+a^{2}\right)^{1 / 2}$ and $x_{0}$ is an initial condition, i.e., $x=x_{0}$ at $x^{\prime}=0$. Here, $x^{\prime}=k_{p}^{-1} d \phi / d \zeta$ and is the normalized axial electric field of the plasma wave, i.e., $x^{\prime}=\hat{E}_{z} \equiv E_{z} / E_{0}$, where $E_{0}=m_{e} c^{2} k_{p} / e$ (sometimes referred to as the cold, nonrelativistic wavebreaking field [5]).

Consider an optimized square pulse train where $a_{n}$ is the amplitude of the $n$th pulse. For the first pulse, Eq. (2) is solved with $a=a_{1}$ and the initial condition $x_{0}=$ $x_{\text {mino }_{0}}=1$. Equation (2) is integrated from the front of the pulse to the back. The optimal pulse length $L_{1}$ is determined by the $\zeta$ distance required to reach maximum potential within the pulse, i.e., $x^{\prime}=0$ and $x=x_{\max _{1}}$. The wake behind the first pulse is given by solving Eq. (2) with $a^{2}=0$ using the initial conditions $x^{\prime}=0$ and $x_{0}=x_{\max _{1}}$. The potential of the wake oscillates between $x_{\max _{1}}$ and $x_{\min _{1}}$. The distance required to reach the minimum potential, $x^{\prime}=0$ and $x=x_{\min _{1}}$, is defined to be one half the nonlinear plasma wavelength, $\lambda_{N_{1}} / 2$. The optimal spacing between the first and second pulse is determined by placing the front of the second pulse at the position in the wake of the first pulse for which $x^{\prime}=0$ and $x=x_{\min _{1}}$. Hence, the optimal spacing between the first and second pulse is some odd multiple of $\lambda_{N_{1}} / 2$. In general, for an optimized square pulse train, it can be shown that the amplitude of the wake behind the $n$th pulse oscillates between $x_{\min _{n}} \leq x \leq x_{\max _{n}}$, where

$$
\begin{gathered}
x_{\min _{n}}=\gamma_{g}^{2}\left[x_{\max _{n}}\left(1+\beta_{g}^{2}\right)-2 \beta_{g}\left(x_{\max _{n}}^{2} / \gamma_{g}^{2}\right)^{1 / 2}\right], \\
x_{\max _{n}=} \gamma_{g}^{2}\left[x_{\min _{n-1}}\left(1+\beta_{g}^{2}\right)\right. \\
\\
\left.-2 \beta_{g}\left(x_{\min _{n-1}}^{2}-\gamma_{\perp_{n}}^{2} / \gamma_{g}^{2}\right)^{1 / 2}\right] .
\end{gathered}
$$

Here, $\gamma_{\perp_{n}}=\left(1+a_{n}^{2}\right)^{1 / 2}$ and $x_{\min _{0}} \equiv 1$. Furthermore, the maximum electric field amplitude behind the $n$th pulse is given by

$$
\hat{E}_{\max _{n}}^{2}=2 \gamma_{g}^{2}\left[x_{\max _{n}}-1 / \gamma_{g}^{2}-\beta_{g}\left(x_{\max _{n}}^{2}-1 / \gamma_{g}^{2}\right)^{1 / 2}\right],
$$

where $\hat{E}_{\max _{n}}=E_{\max _{n}} / E_{0}$. In deriving Eqs. (3)-(5), the spacing between pulses and the pulse lengths are assumed to be optimized, such that the $n$th pulse begins at $x=x_{\min _{n-1}}$ and ends at $x=x_{\max _{n}}$. Both the optimal width $L_{n}$ of the $n$th pulse and the nonlinear wavelength of the wake behind the $n$th pulse (and, hence, the optimal spacing between pulses) increase with increasing $n$. Wave breaking occurs when the electron fluid velocity becomes equal to the plasma-wave phase velocity $v_{g}$. When this occurs, the electron fluid density becomes singular. From Eq. (1), wave breaking occurs when $x_{\min _{n}} \rightarrow 1 / \gamma_{g}$, which implies $x_{\max _{n}} \rightarrow x_{\mathrm{WB}}=\left(2 \gamma_{g}^{2}-1\right) / \gamma_{g}$. This corresponds to a wave-breaking electric field of $\hat{E}_{\mathrm{WB}}^{2}=2\left(\gamma_{g}-1\right)$, or $E_{z}=E_{\mathrm{WB}}[4]$.

The above results, i.e., Eqs. (3)-(5), are valid for laser pulses with arbitrary group velocities $v_{g} \leq c$. Subluminous group velocity effects, $v_{g}<c$, become important at high plasma densities, since $\gamma_{g} \simeq \omega / \omega_{p} \sim 1 / n_{e 0}^{1 / 2}$. 
In the limit $v_{g}=c$, Eqs. (1)-(5) simplify significantly [15-17]. Numerical solutions to Eq. (1) indicate that for $x^{2} \ll x_{\mathrm{WB}}^{2}$ and $\gamma_{g}^{2} \gg 1$, Eq. (1) can be approximated by the limit $\beta_{g} \rightarrow 1$, i.e., $[7,8]$

$$
2 x^{\prime \prime}=\gamma_{\perp}^{2} / x^{2}-1,
$$

where the prime denotes $k_{p}^{-1} d / d \zeta$. For a series of optimized square pulses, analytic solutions can also be readily obtained from this reduced equation. In particular,

$$
\begin{aligned}
& x_{\max _{n}}=\gamma_{\perp_{1}}^{2} \gamma_{\perp_{2}}^{2} \cdots \gamma_{\perp_{n}}^{2}, \\
& \hat{E}_{\max _{n}}=x_{\max _{n}}^{1 / 2}-x_{\max _{n}}^{-1 / 2},
\end{aligned}
$$

and $x_{\min _{n}}=1 / x_{\max _{n}}$. Furthermore, the optimal width of the $n$th pulse $L_{n}$ and the nonlinear wavelength of the wake behind the $n$th pulse, $\lambda_{N_{n}}$, are given by

$$
\begin{gathered}
L_{n}=\left(2 / k_{p}\right) x_{\max _{n}}^{1 / 2} E_{2}\left(\rho_{n}\right), \\
\lambda_{N_{n}}=\left(4 / k_{p}\right) x_{\max _{n}}^{1 / 2} E_{2}\left(\hat{\rho}_{n}\right),
\end{gathered}
$$

where $E_{2}$ is the complete elliptic integral of the second kind, $\rho_{n}^{2}=1-\gamma_{\perp_{n}}^{2} x_{\max _{n}}^{-2}$ and $\hat{\rho}_{n}^{2}=1-x_{\max _{n}}^{-2}$. The optimal spacing between the end of the $n$th pulse and the front of the $n$th +1 pulse is an odd integer multiple of $\lambda_{N_{n}} / 2$. Note for equal pulse amplitudes, i.e., $a_{1}=a_{2}=$ $\cdots \equiv a_{0}, x_{\max _{n}}=\gamma_{\perp_{0}}^{2 n}=\left(1+a_{0}^{2}\right)^{n}$. In the limit $x_{\max _{n}}^{2} \gg$ $1, k_{p} L_{n} \simeq 2 \gamma_{\perp_{0}}^{n}, k_{p} \lambda_{N n} \simeq 4 \gamma_{\perp_{0}}^{n}$, and $x_{\max _{n}}^{\prime} \simeq k_{p} \gamma_{\perp_{0}}^{n}$. Several recent papers have addressed various aspects of this problem [15-17].

The maximum normalized electric field, $\hat{E}_{\max }=$ $x_{\max }^{\prime}=E_{\max } / E_{0}$, for an optimized train of $n$ square pulses of equal amplitudes, is plotted in Fig. 1 versus the quantity $a_{T}^{2} \simeq n a_{0}^{2}$, using the above analytical results. For $\gamma_{g} \gg 1$ and $x^{2} \ll x_{\mathrm{WB}}^{2}, \hat{E}_{\max }$ is approximately independent of $n_{e 0}$. The curves show the result for 1, 3, 5, 10, and 100 pulses. Figure 1 indicates that just a few optimized square pulses are far more efficient than a single pulse. For example, at $n_{e 0}=10^{15}$

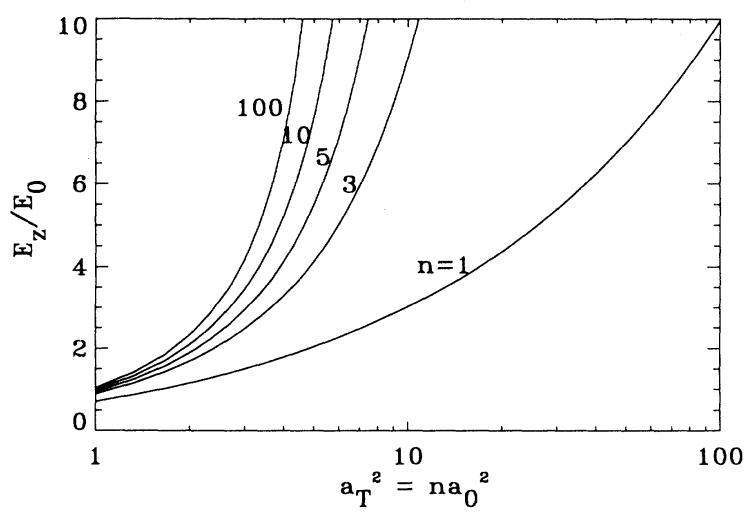

FIG. 1. The maximum electric field $\left(\hat{E}_{\max _{n}}\right)$ vs the quantity $a_{T}^{2}=n a_{0}^{2}$ for $n=1,3,5,10$, and 100 .

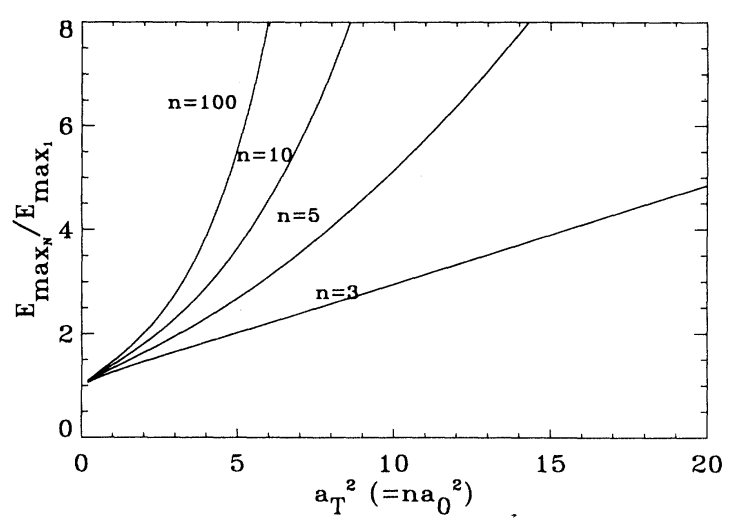

FIG. 2. The ratio of the maximum field achieved with a train of pulses $\left(\hat{E}_{\max _{n}}\right)$ over that achieved with a single pulse $\left(\hat{E}_{\max _{1}}\right)$ of the same energy versus the quantity $a_{T}^{2}=n a_{0}^{2}$ for $n=3,5,10$, and 100 .

$\mathrm{cm}^{-3}\left(\lambda=1 \mu \mathrm{m}, \gamma_{g} \simeq 10^{3}, E_{\mathrm{WB}} \simeq 1.3 \mathrm{GV} / \mathrm{cm}\right)$, three square pulses can be used with an intensity $I=3.5 \times 10^{18}$ $\mathrm{W} / \mathrm{cm}^{2} /$ pulse $\left(a_{0}^{2}=1.3\right)$ and a total pulse train fluence of $I \tau_{\text {tot }}=27 \mathrm{MJ} / \mathrm{cm}^{2}$ to produce $E_{z}=0.1 \mathrm{GV} / \mathrm{cm}$. Here, $\tau_{\text {tot }}$ is the sum of the pulse durations in the train and $2.7 a_{0}^{2} \simeq 10^{-18} \lambda^{2}[\mu \mathrm{m}] I\left[\mathrm{~W} / \mathrm{cm}^{2}\right]$. A single pulse at $n_{e 0}=$ $10^{15} \mathrm{~cm}^{-3}$ requires $I=3.2 \times 10^{19} \mathrm{~W} / \mathrm{cm}^{2}\left(a_{0}^{2}=12\right)$, over an order of magnitude higher intensity than in each pulse in the train, and a total fluence six times greater $\left(I \tau_{\text {tot }}=130 \mathrm{MJ} / \mathrm{cm}^{2}\right)$, to produce this same $E_{z}$. (A low density was chosen for this example so that finite-risetime effects could be neglected, as discussed Sec. III B 1.) Figure 1 indicates that the amplitude-efficiency advantage of multiple pulses increases with increasing number of pulses $n$ or total laser intensity $a_{0}^{2}$. Figure 2 shows the ratio of the maximum field achieved with a train of pulses $\left(\hat{E}_{\max _{n}}\right)$ over that achieved with an equivalent-energy single pulse $\left(\hat{E}_{\text {max }_{1}}\right)$ versus $a_{n}^{2}$, demonstrating the energy efficiency of the RLPA as compared with the LWFA.

\section{NUMERICAL OPTIMIZATION}

\section{A. Square pulses}

Figure 3 shows an example of an optimized square pulse train $\left(n=4, a_{0}=1.2, n_{e 0}=10^{16} \mathrm{~cm}^{-3}\right)$, as obtained by a numerical solution of Eq. (1), in which the widths and spacing between pulses are varied in order to maximize $x_{\max }$. For numerical reasons, we used nonideal square pulses that have small but finite rise times, which is valid - as will be explained in Sec. III B 1 - in the limit of low density, as was used in the example of Fig. 3. It is found that $E_{z}=0.56 \mathrm{GV} / \mathrm{cm}$ for $I \tau_{\text {tot }}=19 \mathrm{MJ} / \mathrm{cm}^{2}$.

The laser pulses are optimally located in the regions where $d \phi / d \zeta>0$. If the laser pulse is located in the region of $d \phi / d \zeta<0$, it will absorb energy from, and reduce the amplitude of, the plasma wave. Likewise, if it is in the region of $d \phi / d \zeta>0$, it will impart energy to, and increase the amplitude of, the plasma wave. Whether or 


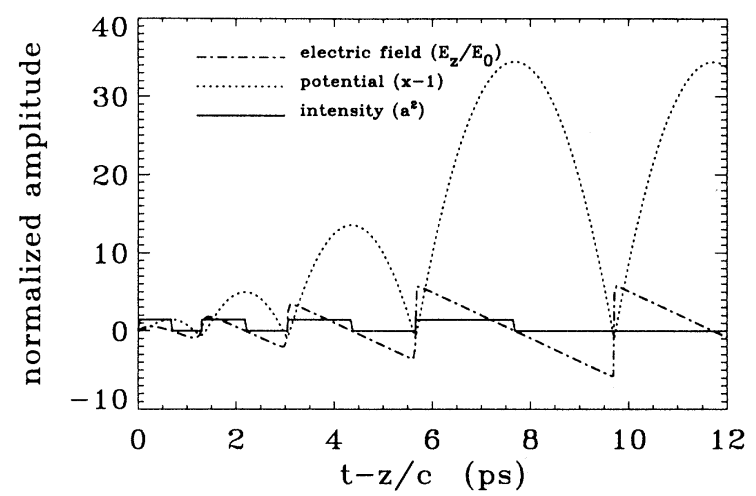

FIG. 3. Numerical solutions for an optimized square pulse train at $n_{e}=10^{16} \mathrm{~cm}^{-3}$ and with $a_{0}=1.2$.

not the laser pulse absorbs energy from or imparts energy to the plasma wave depends on the sign of $d \eta_{R} / d \zeta$, where $\eta_{R}$ is the index of refraction. In the limit $v_{g}=c$, the 1D nonlinear index of refraction for an intense laser pulse in a plasma is given by $[8,21,23]$

$$
\eta_{R} \simeq 1-\left(\omega_{p}^{2} / 2 \omega^{2}\right) /(1+\phi) .
$$

When $d \eta_{R} / d \zeta<0$ (i.e., $d \phi / d \zeta<0$ ), the pulse photons will frequency-up-shift as they propagate; hence, the pulse absorbs energy from the wave [24,23]. Frequency down-shifting (giving energy to the plasma wave) requires $d \eta_{R} / d \zeta>0$ (i.e., $d \phi / d \zeta>0$ ). Hence, to enhance the plasma-wave amplitude, pulses are optimally placed where $d \phi / d \zeta>0$.

When a train that is not optimized is used, for instance fixed interpulse spacings (as in the case of the PBWA), $x_{\max }$ reaches some saturated value before being driven down by destructive interference when the pulses become out of phase with the wave, i.e., when they are located in regions where $d \phi / d \zeta<0$. This is referred to as resonance detuning. Within the optimal (absorption) region, the plasma wave is driven most effectively near $\phi=\phi_{\min }$ (where both the fluid velocity and density of electrons are maximum), and least effectively as $\phi \rightarrow \phi_{\max }$.

\section{B. Sine pulses}

The above results are valid in the limits of either infinitesimally short rise times, or low density. In practice, the rise time $\tau_{\text {rise }}$ of a pulse directly out of a laser is finite and determined by the bandwidth of the laser amplifiers; e.g., currently, the minimum amplified pulse width is $\tau_{\min } \simeq 50 \mathrm{fs}$ [25]. In order to study the effects of plasma density and finite rise times on efficiency, we now consider pulses with an envelope profile $a(\zeta)$ given by a half-period of a sine function. (That Gaussian profiles give qualitatively similar results is verified in other simulations.)

In Fig. 4(a), we plot the wake field resulting from single-pulse excitation (LWFA) including fast oscillations of the laser pulse. For this example, $n_{e 0}=10^{16}$ $\mathrm{cm}^{-3}, a_{0}=1.2$, and the pulse is linearly polarized, i.e.,
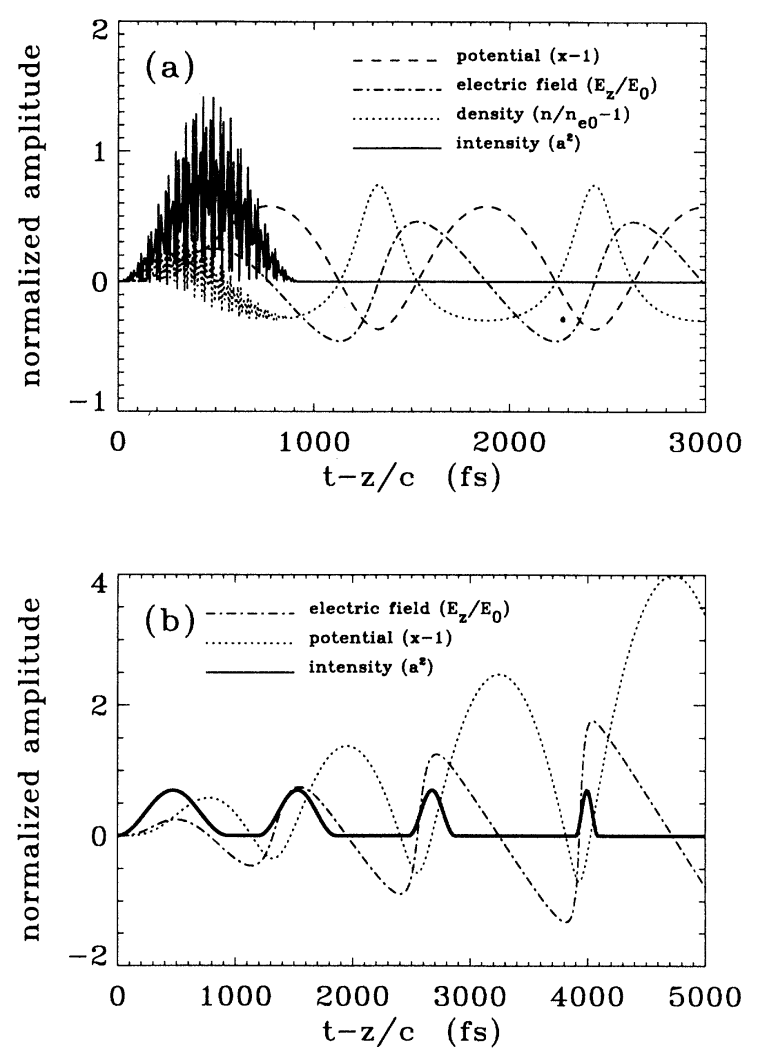

FIG. 4. Numerical solutions for LWFA and RLPA with sine-shaped pulses: (a) single sine pulse at $n_{e}=10^{16} \mathrm{~cm}^{-3}$ with $a_{0} \simeq 1.2$ and (b) an optimized sine pulse train at $n_{e}=10^{16} \mathrm{~cm}^{-3}$ with $a_{0}=1.2$.

$1.4 a_{0}^{2} \simeq 10^{-18} \lambda^{2}[\mu \mathrm{m}] I\left[\mathrm{~W} / \mathrm{cm}^{2}\right]$. The high-frequency density fluctuation inside the laser pulse envelope is due to a fast component of the ponderomotive force at twice the laser frequency, i.e., $a^{2}=\left(\hat{a}^{2} / 2\right)(1+\cos 2 k \zeta)$ for $a=\hat{a} \cos k \zeta$. Figure $4(\mathrm{~b})$ shows an example of a sine pulse train that was optimized numerically. For the laser amplitude, only the envelope, averaged over the fast oscillations, is shown. For this pulse train, $n=4, a_{0}=1.2$, $n_{e 0}=10^{16} \mathrm{~cm}^{-3}$, and the pulses are linearly polarized. The first pulse in Fig. 4(b) has an optimum pulse width $\tau=\tau_{\mathrm{opt}}=940 \mathrm{fs}$ (resonant with $n_{e 0}=10^{16} \mathrm{~cm}^{-3}$ and $a_{0}=1.2$ ) and the final pulse has $\tau=\tau_{\text {opt }}=\tau_{\text {min }} \simeq 200$ fs $\left(I \tau_{\text {tot }}=2.2 \mathrm{MJ} / \mathrm{cm}^{2}\right)$, which gives $E_{z}=0.18 \mathrm{GV} / \mathrm{cm}$ $(\epsilon=0.07)$. As in the square wave case, $\lambda_{N_{n}}$, and thus the spacing between pulses, increases with each succeeding pulse as $x_{\max }$ increases.

\section{Plasma-wave phase resonance region}

Note that whereas with increasing $x_{\max }, \tau_{\text {opt }}$ for succeeding square wave pulses increases $\tau_{\text {opt }} \sim \lambda_{N_{n}} / c$, the opposite is true for multiple sine pulses. This difference arises because, whereas for square pulses $\tau$ is independent of $\tau_{\text {rise }}$, for sine pulses $\tau \simeq 2 \tau_{\text {rise }}$. It is more advantageous to have a short sine pulse width $\left(\tau \ll \lambda_{N_{n}} / c\right)$, so that the highest pulse amplitude is reached near $\phi_{\min }$ (where 
it is most effective in driving the plasma wave), than to have a long sine pulse width $\left(\tau \simeq \lambda_{N_{n}} / c\right)$, so that the pulse is driving the wave for a longer time, albeit mostly when it is less effective (away from $\phi_{\min }$ ). Sine pulses are found to be more effective than square pulses for this same reason. For the later sine pulses, $\tau_{\text {opt }}$ is found to be approximately given by the width of the region between where $\phi<0$ and $d \phi / d \zeta>0$, which defines a "phase resonance width" $L_{\text {res }}$ for finite-rise-time pulses (see Fig. 5). The physical origin of $L_{\text {res }}$ is that in this region (i) the ponderomotive force of the laser pulse is in the right phase with the electron motion to give energy to the plasma wave and (ii) the density of electrons with which the laser pulse can interact is highest. The latter is clearly seen in Fig. 6, which is the same as Fig. 4(b), except the plasma-wave density is plotted instead of the electric field.

For the wake behind the $n$th pulse, $L_{\text {res }}$ can be determined from Eq. (1) in the limit $v_{g}=c$,

$$
L_{\mathrm{res}}=\left(2 / k_{p}\right) x_{\max _{n}}^{1 / 2}\left|E_{2}\left(\pi / 2, \hat{\rho}_{n}\right)-E_{2}\left(\alpha_{1}, \hat{\rho}_{n}\right)\right|
$$

where $\hat{\rho}_{n}^{2} \sin ^{2} \alpha_{1}=1-x_{\max _{n}}^{-1}$. In the limit $x_{\max _{n}} \gg 1$, $L_{\text {res }} \rightarrow k_{p}^{-1} x_{\max _{n}}^{-1 / 2} \simeq 1 / \hat{E}_{\max _{n}}$ and, hence, the resonance becomes sharper with increasing plasma-wave amplitude $\left(Q \equiv \lambda_{N n} / L_{\text {res }} \sim x_{\max _{n}}\right)$.

Figure 7 shows a plot of $L_{\text {res }} / c$, which approximates $\tau_{\text {opt }}$, versus $\epsilon$, where $\epsilon=E_{z} / E_{\mathrm{WB}}$, for various densities. Notice that, in the regime of high $n_{e 0}$, finite-rise-time effects become important at high $\epsilon$, i.e., $\tau_{\text {opt }}$ decreases below $\tau_{\min }$ as $\epsilon$ increases beyond a critical value (e.g., $L_{\mathrm{res}} / c<50$ fs for $\epsilon=0.16$ at $n_{e 0}=10^{16} \mathrm{~cm}^{-3}$ ). Since pulses with $\tau<\tau_{\min } \simeq 50$ fs cannot currently be produced, the later pulses in a train will not be optimized. Although the later pulses with $\tau=\tau_{\min }>\tau_{\text {opt }}$ will continue to increase $\epsilon$, they will do this less effectively than a train in which all pulses are of optimal widths. In fact, a pulse train in this high- $n_{e 0}$ regime can be less amplitude efficient than a single optimized pulse at the same density; i.e., a greater $I \tau_{\text {tot }}$ is required for the pulse train to achieve a given $E_{z}$ at fixed $n_{e 0}$. But, as will be shown in Sec. III B 4, the reduction in efficiency for pulses with

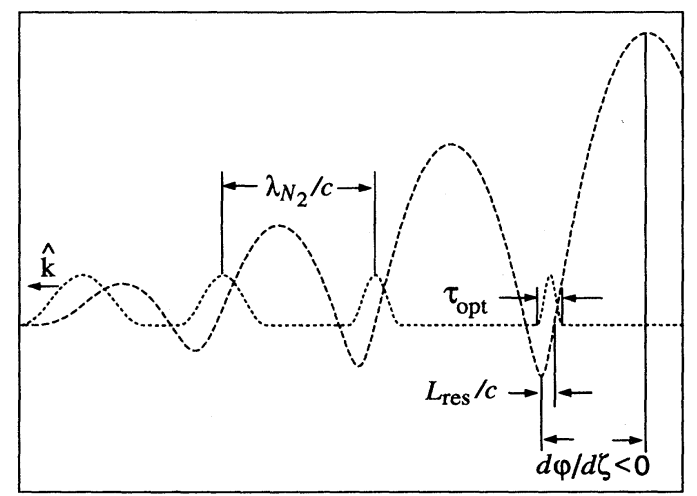

FIG. 5. Legend explaining the definitions of various optimization parameters.

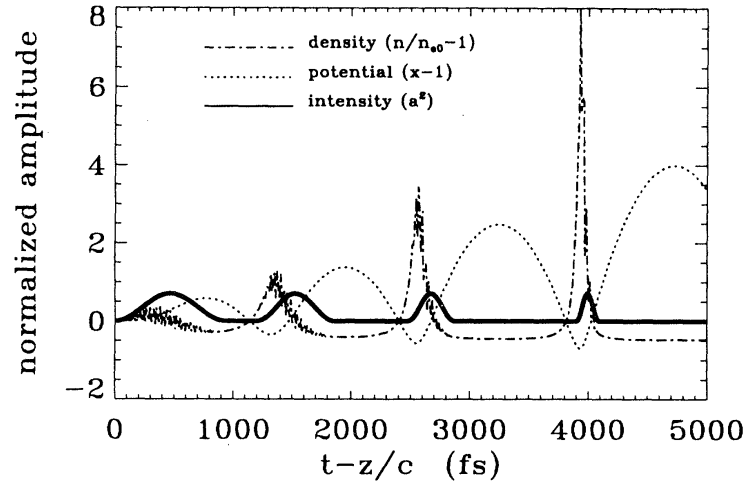

FIG. 6. Numerical solutions for the RLPA with sine-shaped pulses at $n_{e}=10^{16} \mathrm{~cm}^{-3}$ and $a_{0}=1.2$, showing plasma-wave density instead of electric field.

longer than optimal $\tau_{n}$ is more than compensated by a reduction in the sensitivity of the wake-field amplitude to changes in $\lambda_{N}$. Furthermore, high $n_{e 0}$ is unfavorable for electron acceleration because of electron-phase detuning, $\Delta W_{t} \sim \epsilon^{2} n_{e 0}^{-3 / 2}$ in the $\hat{E}_{z}^{2} \gg 1$ and $\gamma_{g}^{2} \gg 1$ regime, as will be discussed in greater detail in the next subsection.

\section{Efficiency comparison between RLPA and LWFA}

Figure 7 indicates that, for low $n_{e 0}$ and up to the previously mentioned critical value of $\epsilon$, the condition $\tau_{\text {opt }} \simeq L_{\text {res }} / c \geq \tau_{\text {min }} \simeq 50$ fs can be satisfied for all of the pulses in a train [as was the case of Fig. 4(b)]. Consequently, multiple sine pulses in this regime are found to be similar to ideal square pulses in that a pulse train is more amplitude efficient than a single pulse at the same density. Specifically, 8 times higher intensity $\left(a_{0}=3.4\right.$, or $\left.I=1.6 \times 10^{19} \mathrm{~W} / \mathrm{cm}^{2}[26]\right)$, corresponding to 2.5 times more fluence $\left(I \tau_{\text {tot }}=5.6 \mathrm{MJ} / \mathrm{cm}^{2}\right)$, is required of a single pulse $\left(\tau=\tau_{\mathrm{opt}}=700\right.$ fs for $\left.n_{e 0}=10^{16} \mathrm{~cm}^{-3}\right)$ to reach the same value of $E_{z}(0.18 \mathrm{GV} / \mathrm{cm})$ as is reached by the train of Fig. 4(b). Reducing the intensity required to

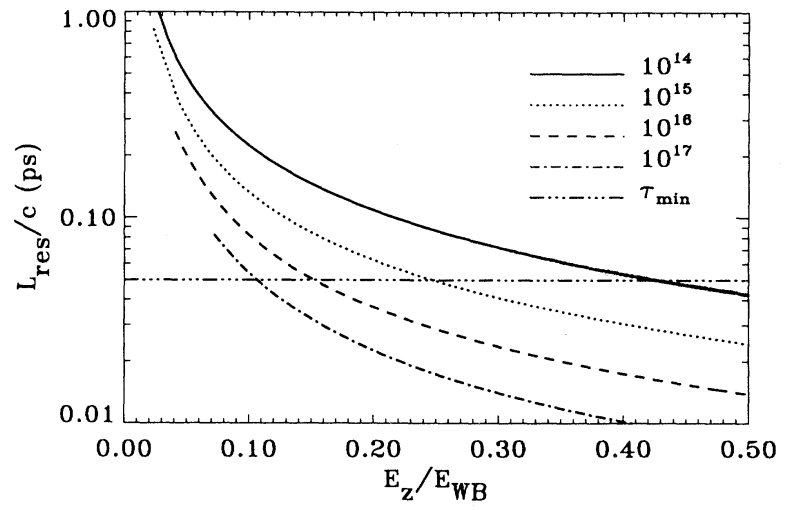

FIG. 7. Plot of $L_{\mathrm{res}} / c$ vs $\epsilon$ for various densities. Finite-rise-time effects are important for $L_{\text {res }} / c<\tau_{\min }$. 
TABLE I. A summary of the various laser, plasma, and acceleration parameters that were found in the comparison between the sine pulse train (first column) and the single sine pulse with the same plasma density (second column) and the single sine pulse with higher density (third column).

\begin{tabular}{llll}
\hline \hline & Train $(4$ pulses $)$ & 1 pulse & 1 pulse \\
\hline Plasma density $n_{e}\left(\mathrm{~cm}^{-3}\right)$ & $10^{16}$ & $10^{16}$ & $10^{18}$ \\
Wave-breaking field $E_{\mathrm{wB}}(\mathrm{GV} / \mathrm{cm})$ & 2.4 & 2.4 & 7.7 \\
Longitudinal field $E_{z}(\mathrm{GV} / \mathrm{cm})$ & 0.18 & 0.18 & 0.18 \\
Plasma wavelength $\lambda_{p}(\mu \mathrm{m})$ & 330 & 330 & 33 \\
Laser field $E_{L}(\mathrm{GV} / \mathrm{cm})$ & 38 & 110 & 22 \\
Laser wavelength $\lambda(\mu \mathrm{m})$ & 1.0 & 1.0 & 1.0 \\
Laser pulse width $\tau_{n}(\mathrm{fs})$ & $940-660-400-200$ & 700 & 90 \\
Laser intensity $a_{0}^{2}$ & $1.4 /$ pulse & 12 & 0.5 \\
Laser intensity $I\left(\mathrm{~W} / \mathrm{cm}^{2}\right)$ & $2 \times 10^{18} /$ pulse & $1.6 \times 10^{19}$ & $7 \times 10^{17}$ \\
Laser power $\left[P \geq I \pi\left(\lambda_{p} / 2\right)^{2}\right](\mathrm{PW})$ & 1.7 & 14 & $6 \times 10^{-3}$ \\
Total laser fluence $\left[I \tau_{\text {tot }}\right]\left(\mathrm{MJ} / \mathrm{cm}^{2}\right)$ & 2.2 & 5.6 & 0.031 \\
Dephasing length $L_{t}(\mathrm{~cm})$ & $2.2 \times 10^{3}$ & $2.2 \times 10^{3}$ & 2.2 \\
Pump depletion length $L_{d}(\mathrm{~cm})$ & $3.0 \times 10^{3}$ & $7.8 \times 10^{3}$ & 40 \\
Total energy gain $\Delta W(\mathrm{TeV})$ & 0.4 & 0.4 & $4.2 \times 10^{-4}$ \\
\hline \hline
\end{tabular}

reach large plasma-wave amplitudes also reduces strongly driven instabilities, such as stimulated Raman scattering, self-focusing, or filamentation, which disrupt either the plasma wave or the laser beam. Pulse-to-pulse phase incoherence of the high-frequency laser oscillations can also reduce instabilities. A single pulse with the same intensity and pulse width as the first pulse in Fig. 4(b), corresponding to 0.43 times the laser fluence $\left(I \tau_{\text {tot }}=2.4\right.$ $\left.\mathrm{MJ} / \mathrm{cm}^{2}\right)$, results in a 3.9 times smaller $E_{z}(46 \mathrm{MV} / \mathrm{cm})$.

In order to drive the same $E_{z}$ with the same $I$ as a sine pulse train, a higher $n_{e 0}$ must be used with a single sine pulse. (Recall that for a single pulse, $E_{z} \sim n_{e 0}^{1 / 2} I$ for $a_{0}^{2}<1$.) Thus, the same value of $E_{z}=0.18 \mathrm{GV} / \mathrm{cm}$ as is reached by the train in Fig. 4(b) is obtained by a less intense single pulse $\left(a_{0}=0.7\right)$ with $\tau=\tau_{\text {opt }}=90 \mathrm{fs}$ at $n_{e 0}=10^{18} \mathrm{~cm}^{-3}$, and with 70 times less energy $\left(I \tau_{\text {tot }}=\right.$ $\left.30 \mathrm{~kJ} / \mathrm{cm}^{2}\right)$. The maximum energy gain, as determined by electron-phase detuning, is $\Delta W_{t}=400 \mathrm{keV}$ for the single pulse. Since energy gain favors low $n_{e 0}$, the pulse train in Fig. 4(b) can accelerate an electron to an energy that is orders of magnitude greater; i.e., $\Delta W_{t}=400$
$\mathrm{GeV}, 1000$ times greater than the single pulse. Thus, a pulse train of equivalent intensity - at either equal or lower $n_{e 0}$-can accelerate an electron to greater energy than a single pulse [27]. Table I gives a summary of the various laser, plasma, and acceleration parameters that were found in the above comparison between the sine pulse train and the single sine pulse. Table II gives the same parameters found in the comparison between the square pulse train and the single square pulse discussed in Sec. III A.

\section{Efficiency comparison between RLPA and PBWA}

Thus far, the RLPA concept has been compared only to the LWFA; in this section, it is compared to the PBWA. In the example of Fig. 8(a), four beat pulses were assumed with amplitudes $a_{0}=1.2$ in a plasma of density $n_{e 0}=10^{16} \mathrm{~cm}^{-3}$. In this case, the unperturbed plasma-wave frequency was used for the beat frequency in a PBWA pulse train, $\Delta \omega \sim \omega_{p}$. However, as expected

TABLE II. A summary of the various laser, plasma, and acceleration parameters that were found in the comparison between the square pulse train and the single square pulse with the same plasma density.

\begin{tabular}{lll}
\hline \hline & Train $(3$ square pulses) & Single pulse \\
\hline Plasma density $n_{e}\left(\mathrm{~cm}^{-3}\right)$ & $10^{15}$ & $10^{15}$ \\
Wave-breaking field $E_{\mathrm{wB}}(\mathrm{GV} / \mathrm{cm})$ & 1.3 & 1.3 \\
Longitudinal field $E_{z}(\mathrm{GV} / \mathrm{cm})$ & 0.1 & 0.1 \\
Plasma wavelength $\lambda_{p}(\mu \mathrm{m})$ & 1000 & 1000 \\
Laser wavelength $\lambda(\mu \mathrm{m})$ & 1.0 & 1.0 \\
Laser pulse width $\tau_{n}(\mathrm{ps})$ & $2-2.5-3.1$ & 4.1 \\
Laser intensity $a_{0}^{2}$ & $1.3 / \mathrm{pulse}$ & 12 \\
Laser intensity $I\left(\mathrm{~W} / \mathrm{cm}^{2}\right)$ & $3.5 \times 10^{18} / \mathrm{pulse}$ & $3.2 \times 10^{19}$ \\
Laser power $\left[P \geq I \pi\left(\lambda_{p} / 2\right)^{2}\right](\mathrm{PW})$ & 27 & 250 \\
Total laser fluence $\left[I \tau_{\text {tot }}\right]\left(\mathrm{MJ} / \mathrm{cm}^{2}\right)$ & 27 & 130 \\
Dephasing length $L_{t}(\mathrm{~cm})$ & $1.1 \times 10^{5}$ & $1.1 \times 10^{5}$ \\
Pump depletion length $L_{d}(\mathrm{~cm})$ & $3.0 \times 10^{4}$ & $1.5 \times 10^{5}$ \\
Total energy gain $\Delta W(\mathrm{TeV})$ & 3 & 11 \\
\hline \hline
\end{tabular}


in this nonlinear regime, resonance detuning between the plasma wave and the PBWA laser train is observed. Therefore, for a more reasonable comparison, the pulse width for the PBWA needs to be optimized for a given plasma density, as was done for the RLPA, but in this case with the constraint that the pulse widths, pulse amplitudes, and interpulse spacings are kept constant for all pulses in the train. The PBWA optimized in this manner is shown in Fig. 8(b). A beat-wave wavelength greater than the one corresponding to the unperturbed density $\lambda_{p}$ is found to be optimum [28], compensating for the increase in the nonlinear wavelength $\lambda_{N}$ that arises from the increase in plasma-wave amplitude. As can be seen from Fig. 8(b), the net effect is to move the spacing between the peaks of the laser pulses closer to $\lambda_{N}$, and thus the locations of the peaks closer to the plasma-wave resonance regions $\left(L_{\text {res }}\right)$. Although the final wake of the optimized PBWA is found in the example of Fig. 8(b) to be similar to that in the RLPA scheme for comparable laser pulse intensities, it should be emphasized that much more energy was required for the former. This is related to the fact that the RLPA is more efficient than the PBWA not only because it mitigates resonance detuning by adjusting to the change in $\lambda_{N_{n}}$ as the plasma wave grows, but because it also adjusts to the change in the phase resonance width, i.e., the plasma wave is driven more efficiently when $\tau_{\text {opt }} \simeq L_{\text {res }} / c$ as in the RLPA than
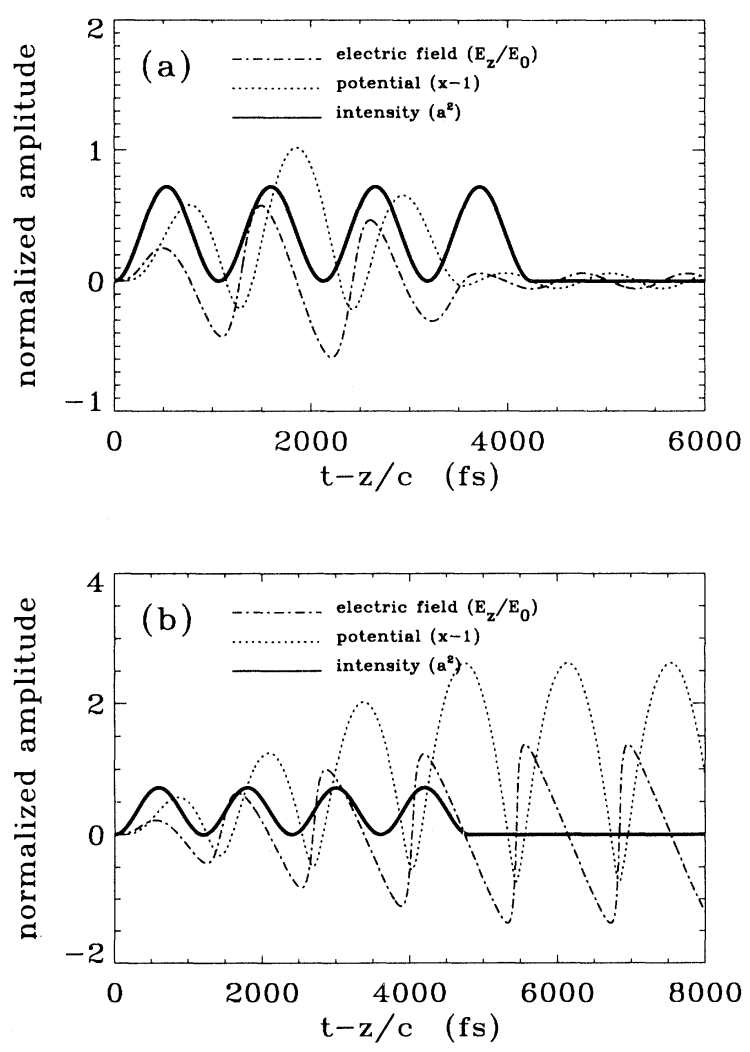

FIG. 8. Numerical solutions for the PBWA: (a) without optimization, showing the effects of detuning and (b) with optimization. when $\tau_{\text {opt }} \simeq L_{n} / c \sim \lambda_{N_{n}} / 2 c$ as in the PBWA.

It is useful to compare the wake fields produced by the various concepts given equal total laser fluence (or energy), since that is the technological limitation imposed by the type of lasers capable of the high intensities required [29]. The intensity and pulse width were varied in such a way that the total laser energy and number of pulses $(n=4)$ were kept the same for both the PBWA and the RLPA. It is found that the optimized PBWA is less energy efficient than either the RLPA or the LWFA for a given density. For example, a PBWA pulse train with $a_{0}=1.0, \tau=1.2 \mathrm{ps}$, where $\tau$ is the pulse width for each pulse, and total fluence in the pulse train equal to $I \tau_{\text {tot }}=3.4 \mathrm{MJ} / \mathrm{cm}^{2}$, produced a normalized wake-field amplitude of $E_{z} / E_{0}=0.4$ at a density of $n_{e 0}=10^{16}$ $\mathrm{cm}^{-3}\left(E_{0} \simeq 96 \mathrm{MV} / \mathrm{cm}\right)$. An equivalent-energy RLPA train $\left(a_{0}=1.6, \tau_{\text {tot }}=1.9 \mathrm{ps}\right)$ gave $E_{z} / E_{0}=3.0$, which is 7.5 times larger. In another example with $n_{e 0}=10^{16}$ $\mathrm{cm}^{-3}$, a LWFA single pulse with $I \tau_{\text {tot }}=5.2 \mathrm{MJ} / \mathrm{cm}^{2}$ $\left(a_{0}=3.4, \tau=700 \mathrm{fs}\right)$ produced a wake larger by a factor of $1.2, E_{z} / E_{0}=1.7$, than an equivalent-energy PBWA (four pulses) with $a_{0}=1.2$ and $\tau=1300 \mathrm{fs}$, which generated $E_{z} / E_{0}=1.4$. These results are summarized in Tables III and IV. Thus, based on the previous discussion, the RLPA is the most energy efficient of all three schemes in this parameter regime.

\section{Wake-field amplitude vs interpulse spacing and pulse width}

The sensitivity of the growth of $\hat{E}_{\max }$ to changes in the pulse widths $\tau$ and interpulse spacings $\lambda_{N_{n}}$ of the laser pulses of Fig. 4(b) $\left(n_{e}=10^{16} \mathrm{~cm}^{-3}\right.$ and $\left.a_{0} \simeq 1.2\right)$ was studied numerically. It is governed by both the number of pulses and the $Q$ of the resonance, where $Q \sim x_{\max }$ is as defined in Sec. III B 1. This can be seen from Fig. 9 in which we plot the maximum electric field $\hat{E}_{\text {max }}$ produced by varying both $\tau$ and $\lambda_{N_{n}}$, for the second $n=2$ (a), third $n=3$ (b), and fourth $n=4$ (c) pulses of the train shown in Fig. 4(b). For instance, from Fig. 9(c), it appears that the fourth pulse $n=4$ is highly sensitive to absolute changes in $\tau$ or $\lambda_{N_{n}}$ in the vicinity of $\tau=\tau_{\text {opt }}$.

It can clearly be seen from Fig. 9(c) that the wake from pulses with $\tau>\tau_{\text {opt }}$ are found to be less sensitive to changes in interpulse spacing than those with $\tau=\tau_{\text {opt }}$, without sacrificing much efficiency. For instance, if the pulse width of the last pulse $(n=4)$ were $\tau=300 \mathrm{fs}$ $\simeq 1.5 \tau_{\text {opt }}$ (instead of $\tau_{\text {opt }}$ ), it is found that a decrease in the optimal spacing between the last and the third pulse $\left(\lambda_{N_{3}}\right)$ by $25 \mathrm{fs}$ (corresponding to $\delta \lambda_{N_{3}} / c \tau_{\text {opt }}=13 \%$ ) results in a decrease of $E_{z}$ (from the value obtained using $\tau=\tau_{\text {opt }}$ and the optimal position) by only $2.2 \%$ (instead of $5 \%)$. Note, in the $\tau=1.5 \tau_{\text {opt }}$ case, $I \tau_{\text {tot }}=2.3$ $\mathrm{MJ} / \mathrm{cm}^{2}$, corresponding to a laser pulse train energy increase of only $4.5 \%$.

The added pulses can also absorb the plasma wave, i.e., the maximum electric field $\left(\hat{E}_{\max _{n}}\right)$ can be reduced to a value below that without it $\left(\hat{E}_{\max _{n-1}}\right)$, when the spacing $\left(\lambda_{n}\right)$ is reduced such that the pulse becomes located in the $d \phi / d \zeta<0$ region. Absorption can be optimized 
TABLE III. A comparison between the RLPA and PBWA at the same plasma density and laser energy fluence shows that the former produces a 7.5 times greater wake field.

\begin{tabular}{lll}
\hline \hline & RLPA & PBWA \\
\hline Plasma density $n_{e}\left(\mathrm{~cm}^{-3}\right)$ & $10^{16}$ & $10^{16}$ \\
Total laser fluence $I \tau_{\text {tot }}\left(\mathrm{MJ} / \mathrm{cm}^{2}\right)$ & 3.4 & 3.4 \\
Laser intensity $a_{0}^{2}$ & $2.6 /$ pulse & $1.0 /$ pulse \\
Laser pulse width $\tau_{n}(\mathrm{fs})$ & $940-540-320-100$ & 1200 \\
Longitudinal field $E_{z} / E_{0}$ & 3.0 & 0.4 \\
\hline \hline
\end{tabular}

just as amplification can, by varying $\tau$ and $\lambda_{N}$, with the maximum amount of absorption equaling the maximum amount of amplification. The second pulse can in fact totally absorb the plasma wave produced by the first pulse [16], the energy of the plasma wave going into up shifting the frequency of the light $[23,24]$.

The wake-field amplitude is less sensitive to an increase in the spacing $\left(\lambda_{n}\right)$, since this moves the pulse further from the $d \phi / d \zeta<0$ region, and thus the wake continues to be enhanced, but less effectively. As $\lambda_{n}$ increases beyond its optimum value, $\hat{E}_{\max _{n}}$ approaches asymptotically the value it had without the pulse, $\hat{E}_{\max _{n-1}}$. Thus, the larger the value of $n$, the less the sensitivity to spacing, since the value of $\hat{E}_{\max _{n-1}}$ is large to begin with, and thus the relative change, $\Delta \hat{E}_{\max _{n}} / \hat{E}_{\max _{n-1}}$ cannot be as large as it is for, say, the $n=2$ pulse, for which $\hat{E}_{\max _{n-1}}=\hat{E}_{\max _{1}}$ is smaller. (See the scaling change of $\hat{E}_{\max _{n}}$ for the three plots of Fig. 9.)

\section{Wake-field amplitude vs plasma density}

Since the exact resonant plasma density is difficult to produce with current technology, we will consider the stability of the final RLPA wake field to variation of the ambient plasma density. In Fig. 10(a), the sensitivity of the wake field versus the ambient plasma density for the pulse train in Fig. 4(b) is shown. The density resonance width is 0.51 , which is defined as $\Delta n_{e} / n_{e 0}=\left(n_{U}-n_{L}\right) / n_{e 0}$, where $n_{U}$ and $n_{L}$ are the upper and lower values of the ambient density for which the wake amplitude is one half of its peak value (the peak value occurs at the resonant ambient density $n_{e 0}$ ). For comparison, the density resonances for the PBWA pulse train of Fig. $8(\mathrm{~b})$ and the LWFA pulse of Fig. 4(a) are shown in Fig. 10(b) and Fig. 10(c), respectively. The arrows indicate the densities corresponding to the resonant densities in the linear

TABLE IV. A comparison between the LWFA and PBWA at the same plasma density and laser energy fluence shows that the former produces a 1.2 times greater wake field.

\begin{tabular}{lll}
\hline \hline & LWFA & PBWA \\
\hline Plasma density $n_{e}\left(\mathrm{~cm}^{-3}\right)$ & $10^{16}$ & $10^{16}$ \\
Total laser fluence $I \tau_{\text {tot }}\left(\mathrm{MJ} / \mathrm{cm}^{2}\right)$ & 5.2 & 5.2 \\
Laser intensity $a_{0}^{2}$ & 11 & $1.4 /$ pulse \\
Laser pulse width $\tau_{n}(\mathrm{fs})$ & 700 & $1300 /$ pulse \\
Longitudinal field $E_{z} / E_{0}$ & 1.7 & 1.4 \\
\hline \hline
\end{tabular}
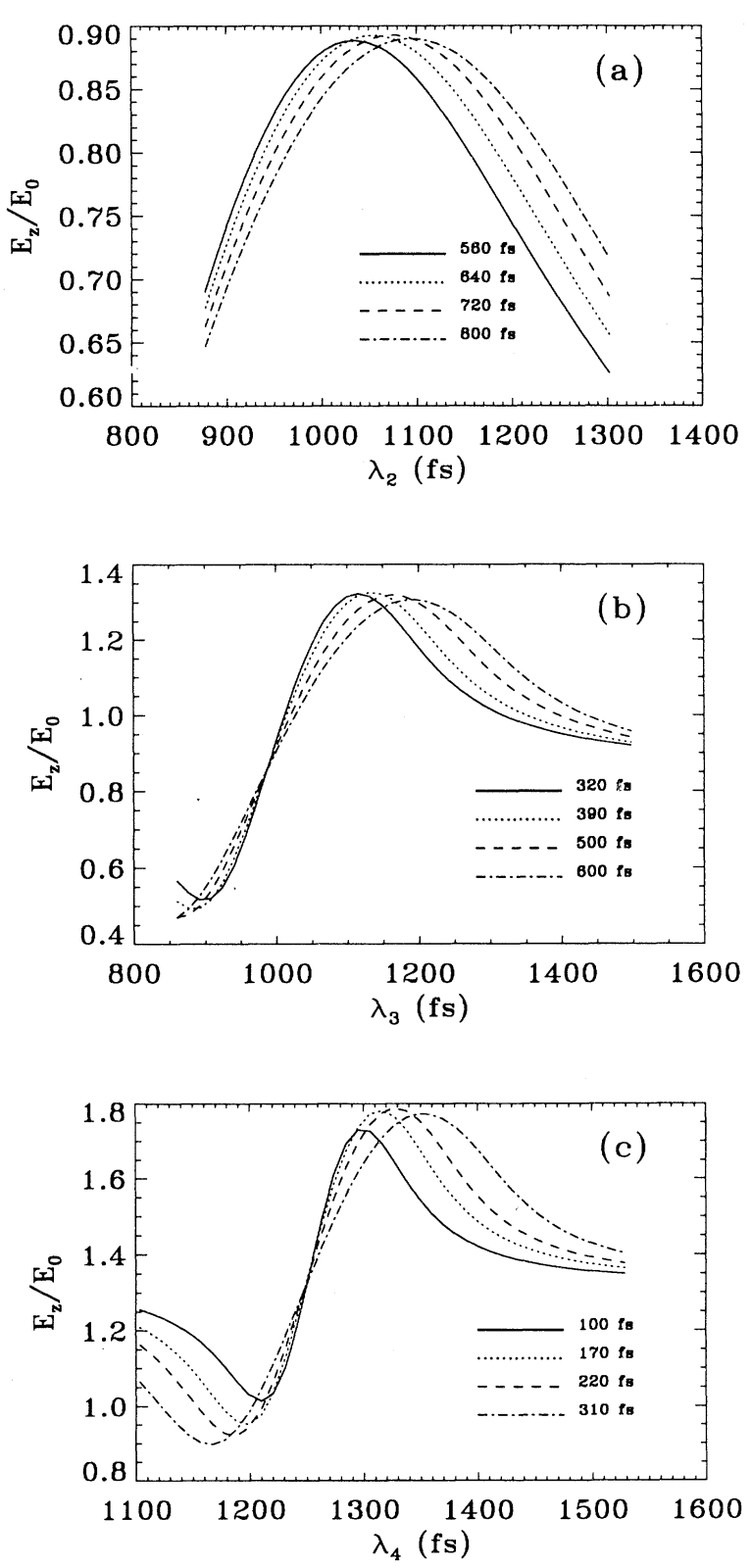

FIG. 9. The maximum electric field $\hat{E}_{\max _{n}}$ produced by varying both the pulse widths $\tau$ and interpulse spacings $\lambda_{N_{n}}$, for the second $n=2$ (a), third $n=3$ (b), and fourth $n=4$ (c) pulses. Note the change in scaling of $\hat{E}_{\max }$ for the three plots. 
approximation, $\Delta \omega=\omega_{p}\left(n_{e}\right)$ for fixed $\Delta \omega$ in the PBWA, and $\tau=2 \pi / \omega_{p}\left(n_{e}\right)$ for fixed $\tau$ in the LWFA. As expected, since it is impulsively driven, the LWFA is found to be the least density sensitive, with a resonance width equal to 3.90. For the PBWA, the corresponding density resonance width is found to be equal to 0.62 . Thus despite the much greater efficiency of the RLPA than the PBWA, their sensitivities to ambient density variation are similar. Achieving a density uniformity meeting this requirement should pose no significant technological challenges-at least for a proof-of-principle experiment-since, in fact, by use of multiphoton ionization [30], uniform laboratory plasmas have been created over distances on the order of $10 \mathrm{~cm}$.
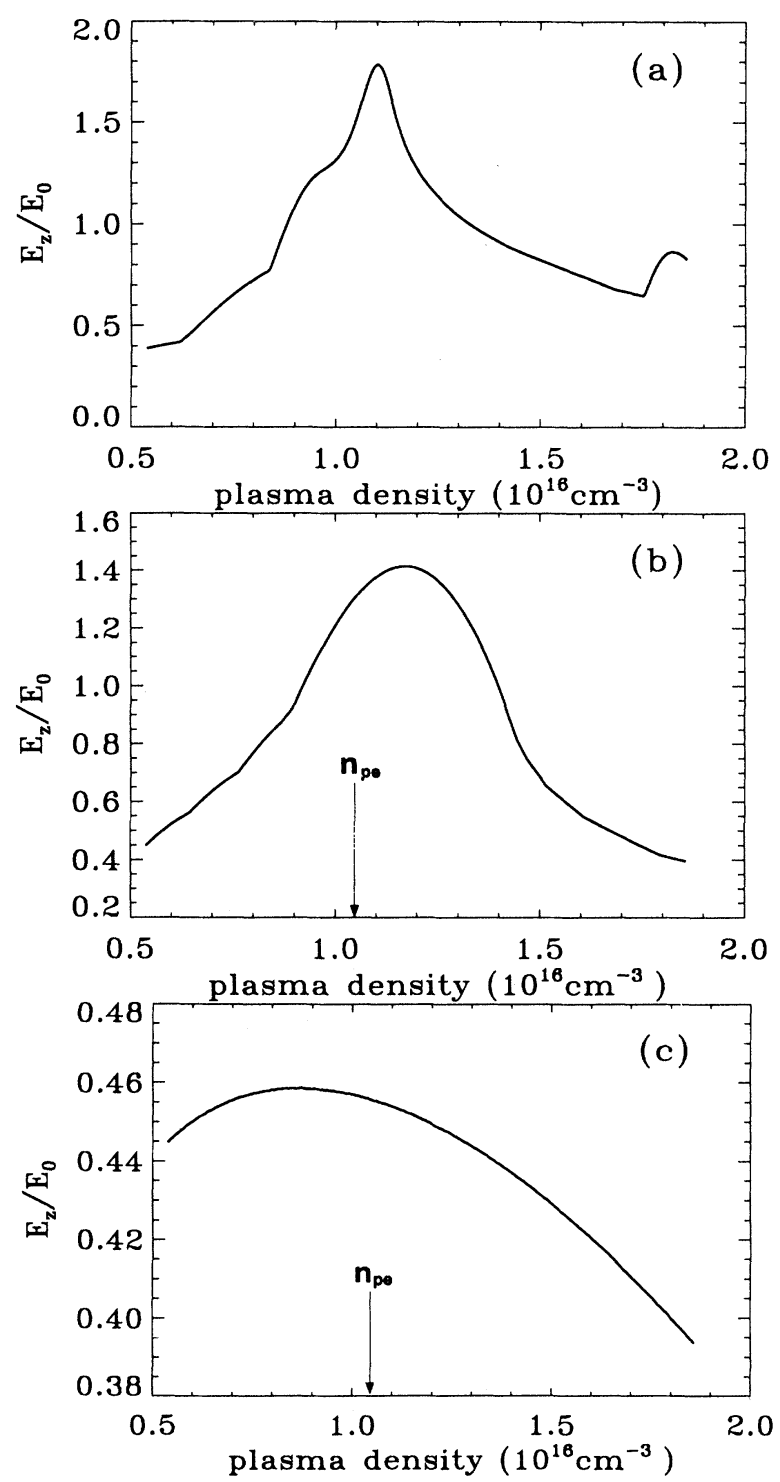

FIG. 10. Final wake-field amplitude as a function of the value of the initial plasma density for: (a) RLPA, (b) PBWA, and (c) LWFA. The arrows indicate the densities corresponding to the resonant densities in the linear approximation, $\Delta \omega=\omega_{p}\left(n_{e}\right)$ for fixed $\Delta \omega$ in the PBWA, and $\tau=2 \pi / \omega_{p}\left(n_{e}\right)$ for fixed $\tau$ in the LWFA.

\section{Wake-field amplitude vs laser intensity}

In addition to density variation, shot-to-shot laser intensity fluctuations can result in detuning. Figure 11(a) shows the dependence of wake-field amplitude on the laser intensity for the RLPA, with the same pulse widths and interpulse spacings as were used in the pulse train shown in Fig. 4(b). As usual we assume here that the intensities of all pulses in the train are the same. Note the multiple peaks and sudden discontinuities in the slope of the curve. They correspond to the various pulses coming in and out of resonance as $\hat{E}_{\max }$ and thus $\lambda_{N}$ change with increasing intensity. The peak at $a_{0}^{2}=1.4$ corresponds to optimization of all pulses. As the intensity $\left(a_{0}^{2}\right)$ increases, the position of the fourth pulse moves toward the absorption region $(d \phi / d \zeta<0)$ and thus $x_{\max }$ becomes reduced. At $a_{0}^{2}=1.6$, the fourth pulse moves into the emission region again $(d \phi / d \zeta>0)$ and there is a sharp discontinuity. Another discontinuity appears at $a_{0}^{2}=2.1$ as the third pulse moves from the absorption to the emission region. The peak at $a_{0}^{2} \sim 2.3$ corresponds to the fourth pulse reaching resonance again. Unlike the RLPA case [Fig. 11(a)], Fig. 11(b) - which shows the sensitivity of the PBWA - does not have several peaks, since the pulses in this case are much longer than $L_{\text {res }}$, and since the intensity in this example was optimized in such a way that
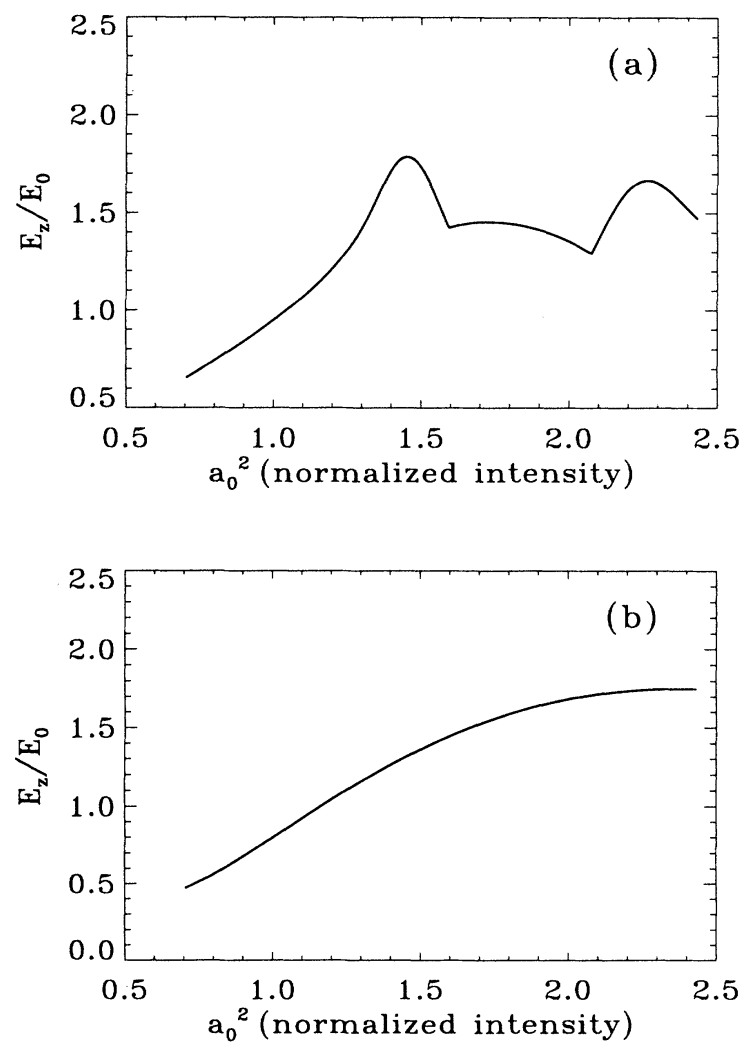

FIG. 11. Final wake-field amplitude as a function only of the laser intensity (constant $\lambda_{n}$ and $L_{n}$ ) for (a) RLPA, and (b) PBWA. 
detuning would not occur. However, as can be seen from Fig. 11(a), the amplitude fluctuations of the RLPA are in the worst case only $20 \%$ for a $10 \%$ change in laser intensity, which does not represent a serious problem since shot-to-shot intensity stabilities of $\leq 5 \%$ are achievable.

\section{PARTICLE-IN-CELL CODE SIMULATIONS}

In order to study kinetic effects, we used a particlein-cell (PIC) code with one spatial dimension and three velocity dimensions. The simulation is fully relativistic and incorporates all of Maxwell's equations. In order to simulate a laser pulse, one boundary becomes an antenna. A sine wave oscillates at the laser wavelength, in this case $1 \mu \mathrm{m}$. To get the correct pulse shape, another sine wave modulates the laser wave so that the pulse has the shape of a half sine-wave equal to the pulse width. A spatial grid was set up so that one laser wavelength was equal to 20 grid points. For a particle density $n_{p}$ of $1.4 \times 10^{16}$ $\mathrm{cm}^{-3}$, similar to that chosen in the previous fluid model simulations, the plasma wavelength $\lambda_{p}$ is $279 \mu \mathrm{m}$, or 940 fs.

The total domain of the simulation is $10 \lambda_{p}$ in length. The electron-ion mass ratio is $1 / 1836$, with the electron charge chosen to give the correct $\lambda_{p}$ for the above $n_{p}$ with 10 particles/cell. The first simulation run, shown in Fig. 12(a), was for a LWFA with a pulse width equal
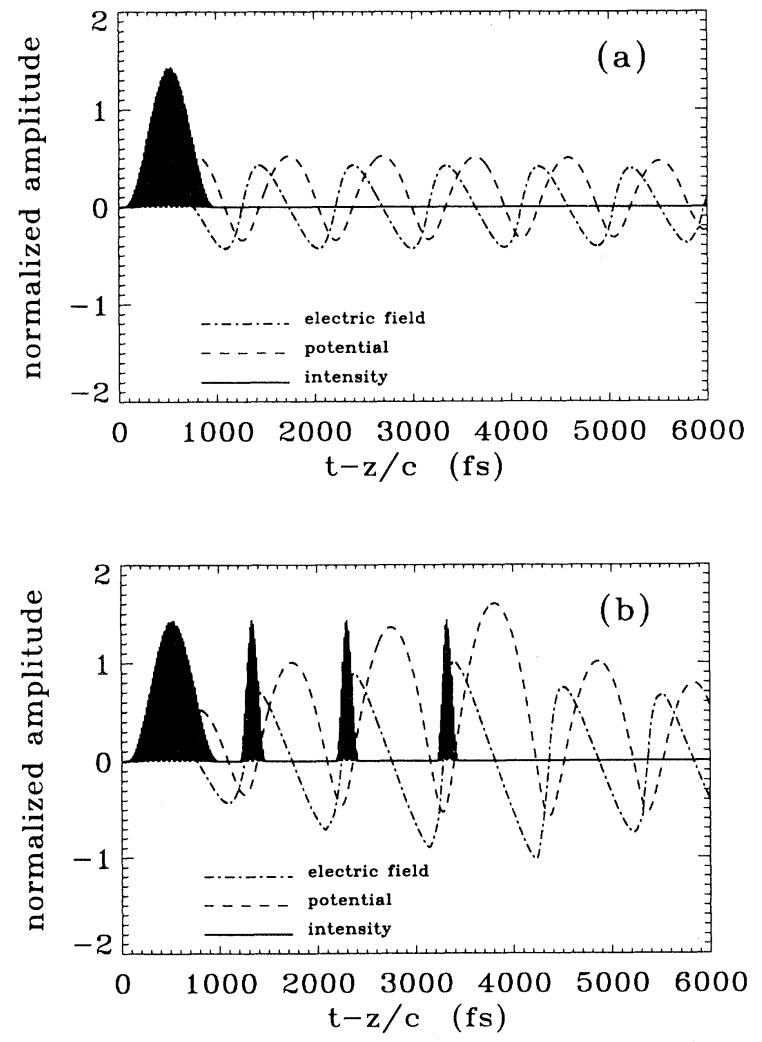

FIG. 12. PIC simulation solution with $n_{e}=10^{16} \mathrm{~cm}^{-3}$ and $a^{2}=1.44$ for (a) a single sine pulse, and (b) four sine pulses. Plasma-wave damping is observed at late times only in the latter case. to $\lambda_{p}$. Qualitatively, it has the same characteristics as that of the fluid model, although the density used in the PIC code was slightly higher. Comparison of these two results validates the quasistatic approximation and the assumption of nonevolving pulse shapes used in the fluid code, but, of course, only for the short distances studied.

Because of the computing expense involved in running the PIC code, optimization of the RLPA could not be done by variation of parameters as was done with the fluid code. A good approximation, however, was made by performing a total of four separate simulations, adding one pulse at a time. The half-width of each succeeding pulse was made to coincide with $L_{\text {res }}$, which was determined by the previous simulation. The results appear in Fig. 12(b).

A feature revealed only when the PIC code is used to model the RLPA is particle trapping and damping of the plasma-wave wake field. Notice that in Fig. 12(b) the wake-field amplitude decreases significantly after it reaches its peak amplitude, i.e., after the last of the four laser pulses is added to the train. This is not observed in PIC simulation of the single-pulse case of Fig. 12(a), which implies that trapping and wave damping only occur when the wake is driven to sufficiently large amplitudes. The PIC-simulation particles start with a small temperature such that their initial velocities are much less than the phase velocity of the wave. However, the latter pump pulses cause some of the background electrons to become trapped in the plasma wave, and these trapped electrons continue to be accelerated in the wake behind the pulse train, depleting energy from the plasma wave, thus causing its amplitude to damp. A phase space diagram of longitudinal electron momentum vs time $\left(p_{z} / m_{e} c\right.$ vs $\left.t-z / c\right)$ from the PIC simulation of the RLPA is shown in Fig. 13. The solid line represents the prediction of the cold fluid theory $[8,21]$, i.e., $p_{z} / m_{e} c=\gamma v_{z} / c$, where

$$
\frac{p_{z}}{m_{e} c}=\frac{\left(1+a^{2}\right)-(1+\phi)^{2}}{2(1+\phi)}
$$

assuming $v_{g} \simeq c$. The solid line was obtained by plotting

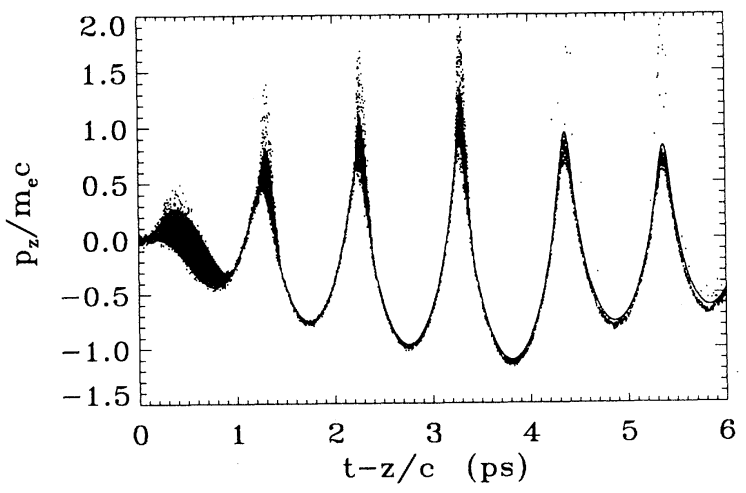

FIG. 13. Phase space diagram of longitudinal momentum vs time $\left(p_{z} / m_{e} c\right.$ vs $\left.t-z / c\right)$ from the PIC simulation of the RLPA. The solid line represents the prediction of the cold fluid theory. 

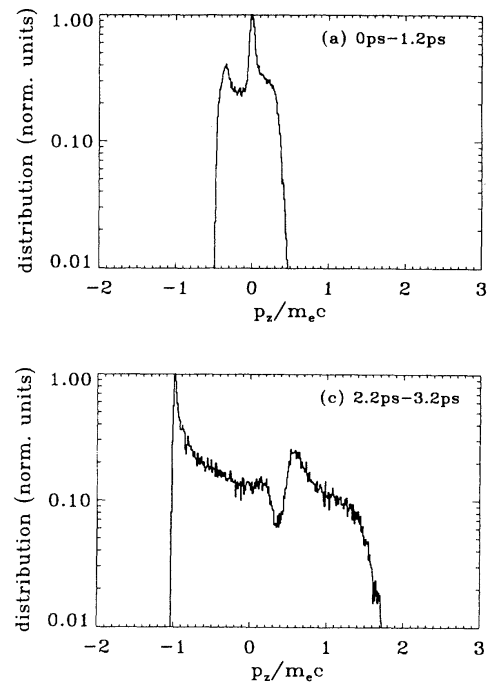
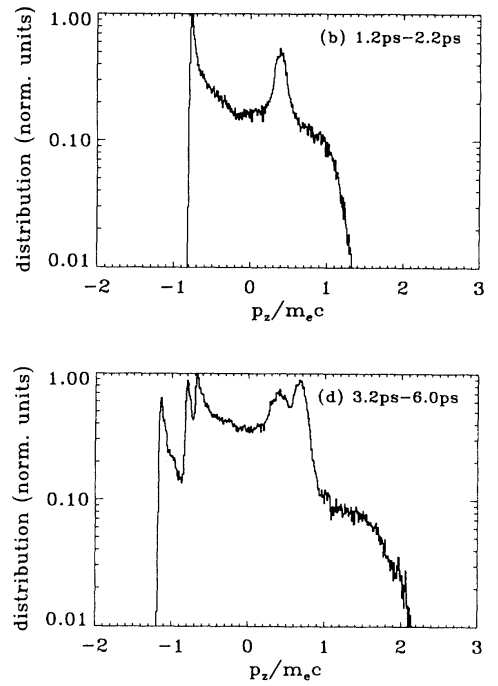

FIG. 14. Normalized electron momentum distribution $\left[f\left(p_{z} / m_{e} c\right)\right.$ vs $\left.p_{z} / m_{e} c\right]$ from the PIC simulation of the RLPA at various times: (a) $t-z / c=0.0-1.2 \mathrm{ps}$, (b) $t-z / c=1.2-2.2$ ps, (c) $t-z / c=2.2-3.2 \mathrm{ps}$, and (d) $t-z / c=3.2-6.0 \mathrm{ps}$. the above expression for $p_{z}(\zeta)$ with both $a^{2}(\zeta)$ and $\phi(\zeta)$ computed by the PIC code. It can be seen from Fig. 13 that the fluid theory and the PIC code agree well for the bulk of the particles. However, an energetic tail of electrons indicates that some trapping by the wave has occurred.

A more quantitative estimate of the fraction of trapped electrons can be obtained from Fig. 14, which shows normalized longitudinal electron velocity distributions $\left[f\left(p_{z} / m_{e} c\right)\right.$ vs $\left.p_{z} / m_{e} c\right]$ calculated at various time intervals (intervals in $t-z / c$ ) during the simulation. These $t-z / c$ intervals correspond roughly to the region between the fronts of the $n$ and $n+1$ pulses and are approximately a plasma wavelength in width (the last interval extends from the end of the last pulse to the end of the simulation region). Hence, for each time interval the momentum distribution is integrated over approximately a plasma wavelength, and in one case, Fig. 14(d), over several $\lambda_{p}$. By comparing Fig. 14 with the fluid theory prediction of Fig. 13, it can be seen that the tail of trapped electrons are those with momenta exceeding approximately $p_{z} / m_{e} c \geq 1$, just to the high-energy side of the peak on the right-hand side in Fig. 14.

\section{PULSE TRAIN GENERATION TECHNIQUES}

There are several ways of producing the required pulse train in practice, but they have yet to be fully investigated in the high-power regime that is required for wakefield generation. The first, shown in Fig. 15, is to use Fourier filtering. In this case, a mask is placed in the pulse stretcher of a chirped pulse amplification system [9] to modify the phase and/or amplitude of every component of the initial pulse in such a way that, when it is recompressed, a series of pulses with arbitrary spacings and widths will be produced [31]. The minimum rise time of each individual pulse is still governed by the gain bandwidth of the amplifiers. This technique has been demonstrated quite effectively in the case of an unamplified pulses using a zero-dispersion stretcher, i.e., the gratings of the stretcher being located at the focal plane of the lenses. The possible difficulties that are encountered with amplification of the pulses are: (1) reduction of the bandwidth due to gain narrowing, (2) distortion of the pulse shapes due to gain saturation, and (3) nonlinear interference between pulses, which overlap in time in the amplifiers when they are stretched. The first problem, gain narrowing, also limits the minimum pulse width of a single pulse, and is overcome by use of larger bandwidth gain media or a combination of amplifiers with different gain media, having adjacent but different central frequencies, effectively producing a larger net bandwidth [32]. The second problem, gain saturation, can be avoided by reducing the single-stage amplification and adding more amplifier stages if necessary. The last problem is circumvented by avoiding any fast amplitude modulation of the chirped pulse in order to minimize nonlinear effects in the amplifier; this implies that phase masks are preferable to amplitude masks. Shaped pulses have already been amplified in the laboratory [33], at least in preliminary ways, but more development is necessary.

By use of either a computer-controlled liquid crystal display [34], or an acoustoptic modulator [35], located in the Fourier plane, the pulses may be modulated in

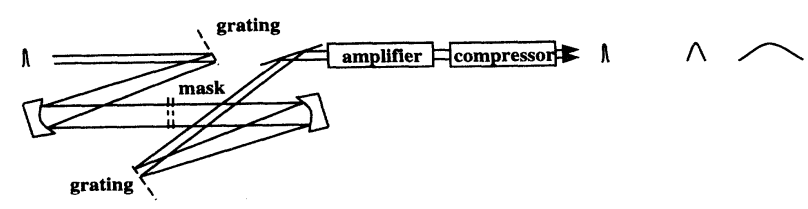

FIG. 15. A variably spaced pulse train with arbitrary pulse widths is produced by use of Fourier filtering in the laser stretcher stage. 
real time (between shots). This provides the possibility of maximizing the wake field experimentally using realtime feedback between the modulator and a diagnostic of the plasma-wave amplitude. A possible problem with the use of spatial filtering with finite resolution is spatial diffraction of the laser beam, the effect of which is to create a spatially dependent temporal pulse profile [36]. However, this is less of a problem for wake-field generation than for other applications of pulse shaping, because the wake field is excited at the laser focus, in the far field, and because it is sensitive to the laser pulse envelope and not changes in the carrier frequency.

A less elegant method of producing optimized pulse trains is to divide the amplified stretched pulse by use of beam splitters placed after the amplifiers, then send the separate pulses to separate compressors, with adjustable lengths and delays, and finally recombine the pulses before they enter the interaction chamber. Alternatively, several pulses could be created using a beam splitter and separate delay lines (as in a Michelson interferometer) placed before the amplifiers, but, as mentioned above, this may create high-frequency beating of the chirped pulses, inducing deleterious effects [37].

The advantage, however, of the pulse-shaping technique discussed in the previous paragraphs is that the pulse widths and interpulse spacings may be tailored independently of each other, unlike the case of optical mixing, as is used in the standard beat-wave accelerator. In the latter case, two long pulses (pulse lengths equal to several plasma wavelengths) and with different central frequencies (differing by the plasma frequency) are amplified in separate laser amplifier chains and then optically mixed in the plasma in order to create a modulation that approximates a train of equally spaced pulses with equal pulse widths (equal to the spacing). As a consequence of both resonance detuning and narrowing of the phaseresonance width $\left(L_{\text {res }}\right)$, the beat-wave method of driving a plasma wave is less efficient then either the RLPA or the LWFA, as discussed in Sec. III B 3.

\section{CONCLUSIONS}

Optimal pulse widths and interpulse spacings were exactly computed from analytical theory for a train of square pulses, and were optimized numerically for a train of sine pulses with realistic rise times. By optimally varying the pulse widths and interpulse spacings, resonance detuning between the laser pulses and the plasma wave can be eliminated. This implies that plasma waves can be driven up to the limits imposed by wave-breaking, particle trapping, and/or the limits of laser pulse train technology.

Resonant regions of the plasma-wave phase space were found where the plasma wave is driven by the laser pulses most efficiently (i.e., the regions where $\phi<0$ and $d \phi / d \zeta>0$ ). In order to overlap the laser pulses with these regions, the optimal interpulse spacings were found to increase as the plasma-wave amplitude (and nonlinear plasma wavelength $\lambda_{N}$ ) increases. One the other hand, the width of this phase resonance region $L_{\text {res }}$ and thus the optimal finite-rise-time laser pulse width $\tau_{\text {opt }}$ - decreases with increasing plasma-wave amplitude, due to wave steepening. It also decreases with increasing background density, in this case due to the relationship $\tau \sim 2 \pi / \omega_{p} \sim n_{e}^{-1 / 2}$, familiar from single-pulse excitation (LWFA).

The sensitivities of the wake field to changes in the plasma density and laser intensity were not found to pose significant technological problems. Wake fields from trains with somewhat-longer-than-optimal pulse widths were found to be considerably less sensitive to variation of interpulse spacing without sacrificing much efficiency.

PIC code results validated the use of the quasistatic approximation and the assumption of nonevolving pulse shapes in the fluid code for the short distances studied. They also showed the importance of particle trapping and wake-field damping for large-amplitude plasma waves.

In these $1 D$ studies, the RLPA was found to have advantages over either the PBWA or the LWFA, since comparable plasma-wave amplitudes may be generated at lower plasma densities, reducing electron-phase detuning, or at lower laser intensities, reducing laser-plasma instabilities. The increased efficiency of the RLPA arises not only because it mitigates resonance detuning by adjusting to the change in $\lambda_{N_{n}}$ as the plasma wave grows, but also because it adjusts to the change in the phase resonance width, i.e., the plasma wave is driven more efficiently when $\tau_{\text {opt }} \simeq L_{\text {res }} / c$ than when $\tau_{\text {opt }} \simeq L_{n} / c \sim$ $\lambda_{N_{n}} / 2 c$ as in the PBWA. This advantage exists even at relatively low plasma-wave amplitudes, far from wave breaking, when the change of $\lambda_{N_{n}}$ is not significant, but the change of $L_{\text {res }}$ is significant.

If large single-stage energy gains are desired $(>100$ $\mathrm{GeV})$, then low plasma densities $\left(n_{e} \leq 10^{16} \mathrm{~cm}^{-3}\right)$ are advantageous because of the favorable scaling of the pump depletion distance, the phase detuning distance, and the phase resonance width. However, in order to reach the required high intensities, and yet remain in the $1 \mathrm{D}$ regime, large laser powers (PW) will be necessary, because of the increase in the plasma wavelength with decreasing density. Such large laser systems will be available within the next few years. In the nearer term, for lower energy-gain applications $(\mathrm{GeV})$, or proof-ofprinciple experiments, higher plasma densities $\left(n_{e} \leq 10^{18}\right.$ $\mathrm{cm}^{-3}$ ) can be used. In this case, much lower laser powers are sufficient (TW), which are currently available from table-top lasers with ultrashort pulses $(\tau \leq 100 \mathrm{fs})$. Practical methods for tailoring laser pulse trains using pulseshaping techniques are also presently available.

\section{ACKNOWLEDGMENTS}

This work was supported by National Science Foundation and the Department of Energy. The authors acknowledge useful conversations with X. Liu, J. Squier, G. Mourou, J. Krall, A. Ting, P. Sprangle, W. Leemans, and W. Mori. 
[1] See e.g., Advanced Accelerator Concepts, edited by J. S. Wurtele, AIP Conf. Proc. No. 279 (AIP, New York, 1993).

[2] T. Tajima and J. M. Dawson, Phys. Rev. Lett. 43, 267 (1979).

[3] C. Clayton et al., Phys. Rev. Lett. 54, 2343 (1985); Y. Kitigawa et al., ibid. 68, 48 (1992); F. Amiranoff et al., ibid. 68, 3710 (1992); H. Hamster et al., ibid. 71, 2725 (1993); C. Clayton et al., ibid. 70, 267 (1994).

[4] A. I. Akhiezer and R. V. Polovin, Zh. Eksp. Teor. Fiz. 30, 915 (1956) [Sov. Phys. JETP 3, 696 (1956)].

[5] J. M. Dawson, Phys. Rev. 113, 383 (1959).

[6] L. M. Gorbunov and V. I. Kirsanov, Zh. Eksp. Teor. Fiz. 93, 509 (1987) [Sov. Phys. JETP 66, 290 (1987)]; P. Sprangle et al., Appl. Phys. Lett. 53, 2146 (1988).

[7] S. V. Bulanov, V. I. Kirsanov, and A. S. Sakharov, Pis'ma Zh. Eksp. Teor. Fiz. 50, 176 (1989) [JETP Lett. 50, 198 (1989)]; V. I. Berezhiani and I. G. Murusidze, Phys. Lett. A 148, 338 (1990); E. Esarey et al., Comments Plasma Phys. Controlled Fusion 12, 191 (1989).

[8] P. Sprangle, E. Esarey, and A. Ting, Phys. Rev. Lett. 64, 2011 (1990); Phys. Rev. A 41, 4463 (1990); A. Ting, P. Sprangle, and E. Esarey, Phys. Fluids B 2, 1390 (1990).

[9] P. Maine et al., IEEE J. Quantum Electron. 24, 398 (1988); G. Mourou and D. Umstadter, Phys. Fluids B 4, 2315 (1992).

[10] P. Sprangle et al., Phys. Rev. Lett. 69, 2200 (1992); J. Krall et al., Phys. Rev. E 48, 2157 (1993); E. Esarey et al., Phys. Fluids B 5, 2690 (1993).

[11] N. E. Andreev et al., Pis'ma Zh. Eksp. Teor. Fiz. 55, 551 (1992) [JETP Lett. 55, 571 (1992)]; T. Antonsen, Jr., and P. Mora, Phys. Rev. Lett. 69, 2204 (1992).

[12] E. Esarey, J. Krall, and P. Sprangle, Phys. Rev. Lett. 72, 2887 (1994)

[13] W. P. Leemans et al., Phys. Rev. A 46, 1091 (1992); P. Monot et al., J. Opt. Soc. Am. B 9, 1579 (1992).

[14] E. Esarey and M. Pilloff, in Advanced Accelerator Concepts, Proceedings of Workshop on Advanced Accelerator Concepts, Lake Geneva, WI, 1994, edited by P. Schoessow (AIP, New York, in press).

[15] D. Umstadter, E. Esarey, and J. Kim, Phys. Rev. Lett. 72, 1224 (1994).

[16] V. I. Berezhiani and I. G. Murusidze, Phys. Scr. 45, 87 (1992).

[17] G. Bonnaud, D. Teychenne, and J. L. Bobin, Phys. Rev. E 48, 3248 (1993); 50, 36 (1994); S. Dalla and M. Lontano, ibid. 49, 1819 (1994); Phys. Lett. A 173, 456 (1993); K. Nakajima, Phys. Rev. A 45, 1149 (1992).

[18] C. G. Durfee III and H. M. Milchberg, Phys. Rev. Lett. 71, 2409 (1993)

[19] D. L. Fisher and T. Tajima, Phys. Rev. Lett. 71, 4338 (1993).
[20] M. D. Perry and G. Mourou, Science 264, 917 (1994).

[21] E. Esarey et al., IEEE Trans. Plasma Sci. PS-21, 95 (1993).

[22] C. D. Decker and W. B. Mori, Phys. Rev. Lett. 72, 490 (1994).

[23] E. Esarey, A. Ting, and P. Sprangle, Phys. Rev. A 42, 3526 (1990).

[24] S. C. Wilks, J. M. Dawson, W. B. Mori, T. Katsouleas, and M. E. Jones, Phys. Rev. Lett. 62, 2600 (1989); V. A. Mironov, A. M. Sergeev, E. V. Vanin, and G. Brodin, Phys. Rev. A 42, 4862 (1990); S. V. Bulanov, I. N. Inovenkov, V. I. Kirsanov, N. M. Naumova, and A. S. Sakharov, Phys. Fluids B 4, 1935 (1992).

[25] J. V. Rudd et al., Opt. Lett. 18, 2044 (1993); J. P. Zhou et al., ibid. 19, 126 (1994); C. P. Barty et al., in Ultrafast Phenomena IX, edited by P. F. Barbara, W. A. Knox, G. A. Mourou, and A. A. Zewail, Springer Series in Chemical Physics Vol. 60 (Springer-Verlag, Berlin, 1994), p. 170.

[26] Current laser technology has achieved a maximum intensity of $I=5.0 \times 10^{18} \mathrm{~W} / \mathrm{cm}^{2}$.

[27] For square pulses, since $\tau_{\text {opt }}$ increases with $x_{\max }$, the appropriate comparison is at fixed $n_{e 0}$; and, in the low- $n_{e 0}$ regime, square pulses with finite rise times $\tau_{\text {rise }} \simeq \tau_{\min } / 2$ behave as ideal square pulses, provided $\tau_{\text {rise }}<L_{\text {res }} / 2 c$ for all the pulses in the train.

[28] C. Tang, P. Sprangle, and R. Sudan, Appl. Phys. Lett. 45, 375 (1984).

[29] For a single pulse with duration $\tau$ less than $10 \mathrm{ps}$, the heat diffusion time, the damage threshold of the last laser grating of a chirped pulse amplification system depends only on total laser fluence. For longer pulses, the damage threshold fluence increases as $\sim \tau^{1 / 2}$. [See, e.g., D. Du et al., Appl. Phys. Lett. 64, 3071 (1994).] The damage threshold of a pulse train with picosecond interpulse spacings behaves similarly, where $\tau$ in this case would be the duration of the pulse train $\tau_{\text {tot }}$.

[30] A. E. Dangor et al., IEEE Trans. Plasma Sci. PS-15, 161 (1987).

[31] A. M. Weiner, J. P. Heritage, and E. M. Kirschner, J. Opt. Soc. Am. B 5, 1563 (1988).

[32] F. G. Patterson, M. D. Perry, and E. M. Campbell, Proc. Soc. Photo-opt. Instrum. Eng. 1040, 160 (1989).

[33] D. Pinkos et al., in Ultrafast Phenomena IX (Ref. [25]), p. 180.

[34] A. M. Weiner, D. E. Leaird, J. S. Patel, and J. R. Wullert II, IEEE J. Quantum Electron. 28, 908 (1992).

[35] C. W. Hillegas, J. X. Tull, D. Goswami, D. Strickland, and W. S. Warren, Opt. Lett. 19, 737 (1994).

[36] J. Paye and A. Migus, J. Opt. Soc. Am. B (to be published).

[37] X. Liu et al., Opt. Lett. (to be published). 